\title{
Genetic modifiers ameliorate endocytic and neuromuscular defects in a model of spinal muscular atrophy
}

\author{
Melissa B. Walsh', Eva Janzen², Emily Wingrove', Seyyedmohsen Hosseinibarkooie ${ }^{2}$, Natalia Rodriguez Muela ${ }^{3}$, \\ Lance Davidow ${ }^{3}$, Maria Dimitriadii, Erika M. Norabuena ${ }^{3}$, Lee L. Rubin ${ }^{3}$, Brunhilde Wirth $^{2}$ and Anne C. Hart ${ }^{{ }^{*}}$ (i)
}

\begin{abstract}
Background: Understanding the genetic modifiers of neurodegenerative diseases can provide insight into the mechanisms underlying these disorders. Here, we examine the relationship between the motor neuron disease spinal muscular atrophy (SMA), which is caused by reduced levels of the survival of motor neuron (SMN) protein, and the actin-bundling protein Plastin 3 (PLS3). Increased PLS3 levels suppress symptoms in a subset of SMA patients and ameliorate defects in SMA disease models, but the functional connection between PLS3 and SMN is poorly understood.

Results: We provide immunohistochemical and biochemical evidence for large protein complexes localized in vertebrate motor neuron processes that contain PLS3, SMN, and members of the hnRNP F/H family of proteins. Using a Caenorhabditis elegans (C. elegans) SMA model, we determine that overexpression of PLS3 or loss of the C. elegans hnRNP F/H ortholog SYM-2 enhances endocytic function and ameliorates neuromuscular defects caused by decreased SMN-1 levels. Furthermore, either increasing PLS3 or decreasing SYM-2 levels suppresses defects in a C. elegans ALS model.

Conclusions: We propose that hnRNP F/H act in the same protein complex as PLS3 and SMN and that the function of this complex is critical for endocytic pathways, suggesting that hnRNP F/H proteins could be potential targets for therapy development.
\end{abstract}

Keywords: PLS3, hnRNP, Neurodegenerative disease, SMN, SMA, Endocytosis

\section{Background}

Neurodegenerative diseases are widespread and usually incurable. The identity of disease-causing genes is frequently known, but the mechanisms and pathways underlying disease onset, progression, and pathology remain elusive. The motor neuron disease spinal muscular atrophy (SMA) is caused by reduced levels of the survival of motor neuron (SMN) protein.

\footnotetext{
* Correspondence: anne hart@brown.edu

'Department of Neuroscience, Brown University, 185 Meeting Street, Mailbox GL-N, Providence, RI 02912, USA

Full list of author information is available at the end of the article
}

Decreased SMN in SMA patients impacts the function of alpha motor neurons, resulting in their dysfunction, degeneration, and consequent muscular atrophy. SMN is required for the biogenesis of small nuclear ribonucleoproteins (snRNPs), which are critical for pre-mRNA splicing [1-3]. SMN is also found in stress granules and other RNP granules in neuronal processes [4-7], and other studies have identified roles for SMN in microRNA biogenesis and endocytic pathways [8-11]. Determining which of these various granules and RNP-related pathways are most pertinent to SMA pathogenesis is critical. To dissect this,

(c) The Author(s). 2020 Open Access This article is licensed under a Creative Commons Attribution 4.0 International License, which permits use, sharing, adaptation, distribution and reproduction in any medium or format, as long as you give appropriate credit to the original author(s) and the source, provide a link to the Creative Commons licence, and indicate if changes were made. The images or other third party material in this article are included in the article's Creative Commons licence, unless indicated otherwise in a credit line to the material. If material is not included in the article's Creative Commons licence and your intended use is not permitted by statutory regulation or exceeds the permitted use, you will need to obtain permission directly from the copyright holder. To view a copy of this licence, visit http://creativecommons.org/licenses/by/4.0/ The Creative Commons Public Domain Dedication waiver (http://creativecommons.org/publicdomain/zero/1.0/) applies to the data made available in this article, unless otherwise stated in a credit line to the data. 
we turn to genetic modifiers of SMA. Understanding why these modifiers suppress SMA-associated defects should reveal pathways critical for SMA pathogenesis.

Plastin 3 (PLS3) was identified as a genetic modifier in type III SMA discordant families, where six asymptomatic individuals had elevated PLS3 levels [12]. PLS3 is a calcium-dependent actin-bundling protein involved in many processes, including cell migration, adhesion, vesicle trafficking, and endocytosis [13, 14]. PLS3 overexpression ameliorates defects in many mouse, zebrafish, and tissue culture models of SMA [9, 15-18]. Because overexpression of PLS3 does not increase SMN protein levels or overtly change SMN localization, PLS3 likely acts with or downstream of SMN to suppress defects in SMA. There is no evidence that PLS3 and SMN proteins directly interact, but both pull down together in a large protein complex that also contains actin [12].

Previous work has demonstrated that decreased SMN function causes endocytic pathway defects. Endocytic defects are seen in C. elegans, mouse, and tissue culture models of SMA [8-10, 19]. These defects are also found in less severe models of SMA where SMN decreases are not sufficient to cause motor neuron loss. PLS3 overexpression suppresses these endocytic pathway defects in tissue culture cells and in an intermediate SMA mouse model, which was generated using antisense oligonucleotides (ASO) targeting SMN [9]. Moreover, two novel PLS3 interacting proteins have been identified and shown to be involved in the endocytosis process: overexpression of coronin 1C (CORO1C)-an F-actin binding and bundling protein-and downregulation of calcineurin-like EF-hand protein 1 (CHP1)-a calcium sensor and inhibitor of calcineurin that collectively dephosphorylates proteins involved in endocytosis-both rescue impaired endocytosis in SMA $[9,19]$.

Another genetic modifier, independently isolated in SMA patient families, also impacts endocytic pathway function. Neurocalcin delta (NCALD) encodes a small $\mathrm{Ca}^{+}$binding protein that acts as a neuronal calcium sensor. Loss of NCALD suppresses defects in mouse, $C$. elegans, and zebrafish SMA models, including the previously described endocytic defects [10]. Combined, the analysis of these four human genetic modifiers suggests that endocytic pathway defects in SMA may be critical contributors to SMA pathogenesis. We hypothesize that the protein complex containing SMN, PLS3, and actin may be critical for endocytic pathway function when SMN levels drop, but our poor understanding of this protein complex hinders progress.

Furthermore, understanding the interaction between PLS3 and SMN could provide insight into other neurodegenerative diseases including ataxia and amyotrophic lateral sclerosis (ALS). Indeed, recently we reported that PLS3 overexpression is beneficial in CHP1-related ataxia in mice that carry biallelic Chp1 mutation [20]. Key proteins have been identified linking ALS and SMA. Several of these proteins function in splicing, mRNP transport, and other processes that overlap with the function of SMN [21-24]. SMN is also known to have a wellestablished interaction with a group of proteins known as GEMINs, and loss of GEMINs is a cellular hallmark of SMA [25]. Interestingly, studies have also shown that Gemin levels are affected in various models of ALS including SOD1 models [26-28]. These studies along with a recently published work that undertook a gene ontology enrichment analysis of published ALS modifier genes found an overlap in shared pathways that may underlie ALS and are also involved in SMA [29]. With evidence that similar pathways and genes are affected across multiple neurodegenerative diseases, it is crucial that one takes into consideration the possibility that a genetic modifier of SMA might also modify ALS.

Therefore, in this study, we focus on understanding the genetic interaction between PLS3 and SMN and testing if similar genetic interactions occur in a C. elegans model of ALS through behavioral analysis. We demonstrate that increased PLS3 expression suppresses locomotion defects in an established C. elegans model of SMA. Through a combination of approaches in C. elegans and vertebrate systems, we identify novel proteins in the PLS3/SMN complex. Using genetic and behavioral tools available in C. elegans, we demonstrate that perturbing the function of these novel proteins modifies defects in SMA models and we identify the hnRNP F/H ortholog, sym-2, as a novel genetic suppressor of smn-1 loss of function defects. We find that PLS3, SMN, and hnRNP $\mathrm{F} / \mathrm{H}$ are in a large protein complex in vertebrate motor neuron processes. Furthermore, we find that similar to PLS3 overexpression, decreasing hnRNP F/H suppresses defects in endocytic pathways caused by reduced SMN levels. Finally, increased expression of PLS3 or decreased expression of hnRNP F/H orthologs suppresses locomotion defects in a C. elegans ALS model [30]. Taken together, these results demonstrate that PLS3, $\mathrm{SMN}$, and hnRNP F/H are functional members of a protein complex that is pertinent to SMA and that the mechanism underlying PLS3 or hnRNP F/H suppression is likely altering endocytic pathway activity. In addition, the suppression of ALS behavioral defects in a C. elegans model by increasing PLS3 or decreasing SYM-2 expression shows that conserved pathways are affected across motor neuron diseases.

\section{Results}

SMN function is essential for proper recovery after exhaustion

The $C$. elegans genome contains a single ortholog of SMN, encoded by the smn-1 gene. The severe loss of 
function allele, smn-1(ok355), has impaired neuromuscular function, and animals usually die during larval development, despite the fact that premature motor neuron death is not observed [31, 32]. A less severe allele, smn-1(cb131), is viable, fertile, and has only subtle defects. smn-1(cb131) is a $\mathrm{D} 27 \mathrm{~N}$ amino acid substitution, analogous to a $\mathrm{D} 44 \mathrm{~V}$ allele found in a type III SMA patient [33, 34]. The locomotion of smn-1(cb131) animals is normal on solid surfaces [35], and we observed no locomotion defects in animals swimming in liquid (assessed as body bends per minute in developmental stage matched animals, Fig. 1b pre-exposure time point, $p=0.746$ ). However, smn1(cb131) animals are resistant to immobilization by pyridostigmine bromide, an acetylcholinesterase inhibitor, suggesting latent defects in cholinergic neuromuscular junction (NMJ) synapse function [35].

Liewald and colleagues showed that channelrhodopsin (ChR2) stimulation of C. elegans cholinergic motor neurons depletes synaptic vesicle pools and results in transiently decreased locomotion post-stimulation [36, 37]. Using this paradigm (Fig. 1a and detailed description in the "Methods" section), we challenged NMJ function in smn-1(cb131) animals. As a result, cholinergic motor neuron stimulation in smn-1(cb131) animals revealed a locomotion defect during recovery after exhaustion, only in the presence of retinal. Before the blue light activation of cholinergic ChR2, locomotion rates of smn-1(cb131) and control animals were indistinguishable. After ChR2 activation for $20 \mathrm{~s}$, locomotion rates decreased in both smn-1(cb131) and control animals. However, the postexhaustion decrease was more profound in smn1 (cb131) animals (locomotion rates were $58 \%$ of controls, Fig. $1 \mathrm{~b}, p=0.001)$. smn-1(cb131) locomotion was restored to control levels $20 \mathrm{~min}$ after stimulation (Fig. 1b, $p=0.869$ ), arguing against neuron or synapse damage caused by ChR2 activation and more likely a delayed recovery after exhaustion in these animals. Thus, we conclude that a latent defect in recovery after exhaustion is revealed in smn-1(cb131) by ChR2 stimulation of cholinergic neuron activity.

To test if smn-1(cb131) defects are caused by diminished SMN-1 levels, we undertook transgenic rescue studies. A single copy of the C. elegans smn-1 gene, including promoter, exons, introns, and 3' untranslated sequences, was inserted into another chromosome by site-directed homologous recombination, creating rtSi10[smn-1p::smn-1] [8]. This transgene restored normal recovery rates after exhaustion in smn-1(cb131); ChR2 animals (smn-1(cb131);rtSi10 versus smn-1(cb131), Fig. 1c, $p=0.043)$. Therefore, diminished levels of SMN1 impair recovery after exhaustion in smn-1(cb131) animals. Next, we used this ChR2 exhaustion assay to assess the impact of PLS3, a previously identified genetic modifier $[12,16]$.

\section{Increased expression of plastin in neurons suppresses} smn-1(cb131) locomotion defects

PLS3 is a protective modifier of SMA; increased PLS3 levels suppress defects in humans and SMA models in zebrafish, flies, and mice $[9,12,16,38-40]$. We determined whether increasing PLS3 levels would ameliorate various functional defects in C. elegans SMA models.

First, we assessed locomotion after ChR2 exhaustion to determine if the introduction of human PLS3 could restore locomotion when SMN-1 function is decreased. We introduced two transgenes; a single-copy transgene (rtSi27[dpy-30p::PLS3]) or a multi-copy transgene (rtIs59 [dpy-30p::PLS3]) was crossed onto the smn-1(cb131);ChR2 background. In both cases, human PLS3 was expressed ubiquitously in somatic tissues using the $d p y$-30 promoter [41]. We found that PLS3 overexpression restored post-exhaustion locomotion rates to normal levels in smn-1(cb131) animals (after cholinergic ChR2 neuron stimulation, Fig. 2a, $p=0.002$ and 0.003). Therefore, we conclude that expression of human PLS3 ameliorates the functional defects caused by decreased SMN-1 in this exhaustion paradigm.

To test for cross-species conservation of plastin function, we also examined the impact of overexpressing the C. elegans PLS3 ortholog, plst-1. Loss of plst-1 exacerbates smn-1 neuromuscular defects in other paradigms [39]. We assessed locomotion in smn-1 mutant animals that ubiquitously express $C$. elegans plst-1 mRNA at increased levels ( $r t E x 850$ [dpy-30p::plst-1]). The multi-copy plst-1 transgene restored normal locomotion rates after exhaustion in smn-1(cb131);ChR2 animals (Fig. 2a, $p=0.006$ ). Combined with previous loss of function studies [39], our results are consistent with the hypothesis that Plastin 3 and its C. elegans ortholog are cross-species modifiers of locomotion defects in SMA models.

SMN is broadly expressed, but its function is required in motor neurons for normal NMJ activity, based on work in mammalian [42] and C. elegans SMA models [31]. Plastin 3 is also broadly expressed in neuronal and non-neuronal tissues [43]. To determine where PLS3 function is required in C. elegans SMA models, we expressed PLS3 in either body wall muscle or neurons of smn-1(cb131) animals. Expression of human PLS3 in neurons (rtEx852[unc-119p::PLS3]), but not in muscles (rtEx851[myo-3p::PLS3]), restored normal recovery of locomotion rates after exhaustion in smn-1(cb131);ChR2 animals (Fig. 2b, $p=0.05$ and $p=0.237$, versus no PLS3, respectively). This is consistent with studies of PLS3 and SMN in vertebrate models, where increasing PLS3 in neurons was also sufficient to ameliorate defects when SMN levels decreased [16]. 


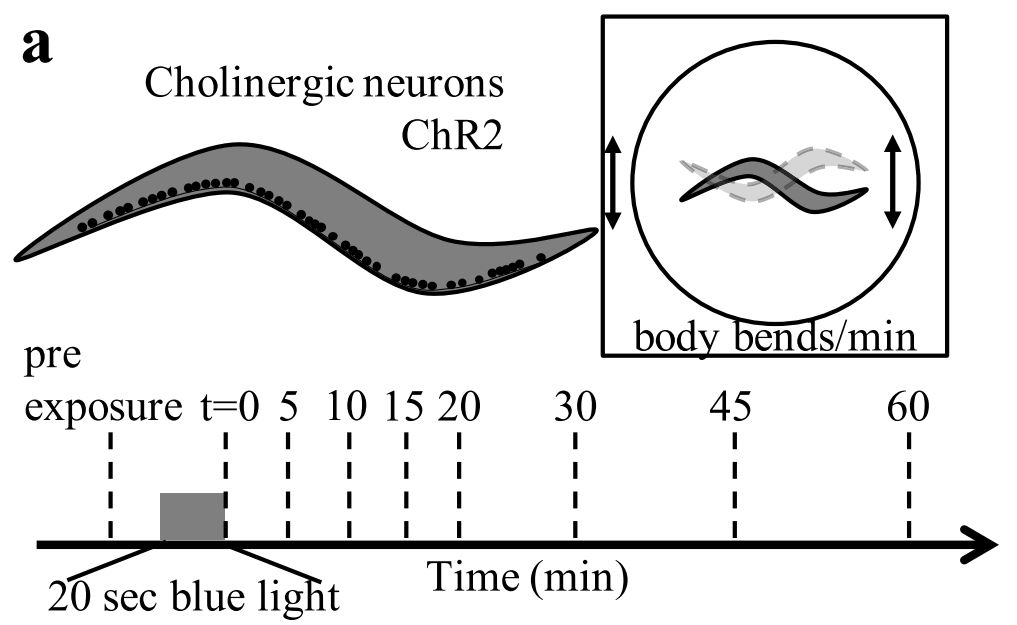

b

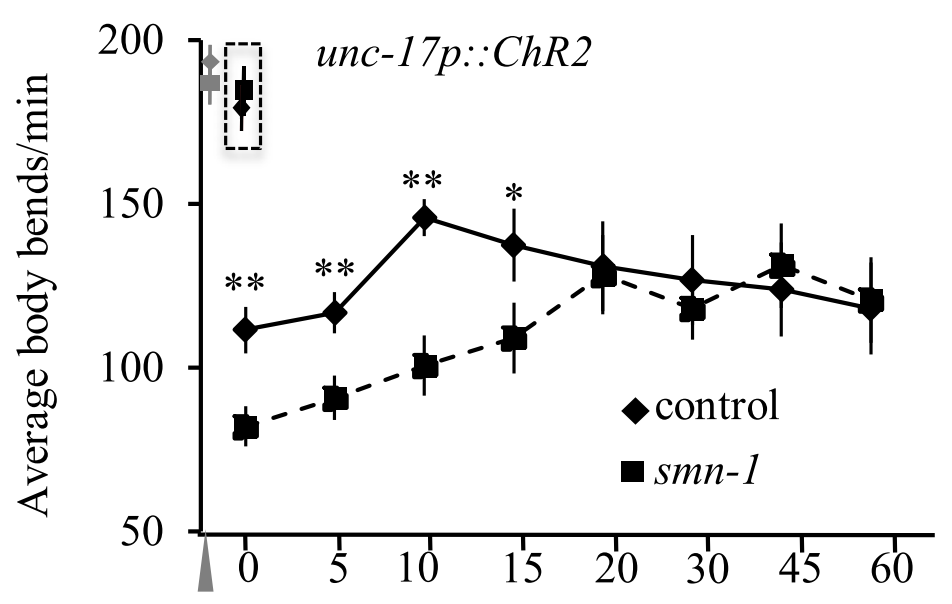

pre-exposure Recovery time (mins)

c

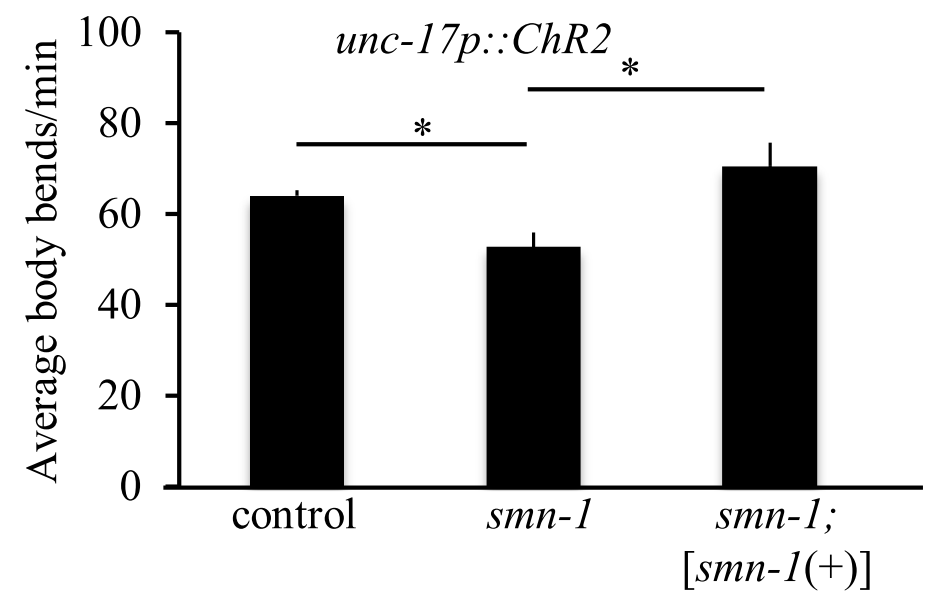

Fig. 1 (See legend on next page.) 
(See figure on previous page.)

Fig. 1 Rescue of smn-1(cb131) exhaustion defects by reintroduction of smn-1(+). a Schematic representation of experimental design. Channelrhodopsin is expressed in cholinergic motor neurons. After overnight retinal feeding, animals are exposed to blue light stimuli for $20 \mathrm{~s}$ in liquid and body bends per minute are recorded over time. A body bend is defined as a muscle contraction resulting in a dorsal to ventral movement across the midline of the animal. b Before blue light stimulation, animals have almost identical locomotion (data points shown in gray). After $20 \mathrm{~s}$ blue light stimulation of channelrhodopsin (ChR2) in cholinergic neurons, smn-1(cb131) partial loss of function animals had decreased locomotion rates when fed retinal and had delayed recovery to original activity. Animals not fed retinal are shown immediately following blue light exposure in a hashed box. Locomotion reported as body bends per minute in liquid. c Introduction of an smn-1 genomic transgene restored normal locomotion rates immediately following blue light illumination. [smn-1(+)] is rtSi10, a homozygous single-copy insertion on chromosome IV (see Additional file 1: Table for details); ANOVA F(3.32, 12.5) =7.27, $p<0.05$. All animals carry oxls364[unc-17p::ChR2] and exhibit no change in locomotion without exogenous retinal feeding (data not shown). The drop in body bends per minute from panel $\mathbf{b}$ to $\mathbf{c}$ is due to methodology. Panel $\mathbf{b}$ is counted manually by eye whereas panel $\mathbf{c}$ and future locomotion rates are calculated using the NABAS program described in the "Methods" section. For all locomotion assays, $n \geq 30$ animals were used per determination and combined from 3 independent and blinded trials. Student's $t$ test: ${ }^{*} p<0.05,{ }^{* *} p<0.01$. SEM indicated

Finally, we examined the impact of increased PLS3 activity in otherwise normal animals. A single-copy insertion of the ubiquitously expression $d p y-30 p:: P L S 3$ transgene had no impact on basal locomotion; activity in liquid was indistinguishable from control animals. However, further increase in PLS3 dosage did alter locomotion. A transgene generated at higher concentrations of $d p y$-30p::PLS3, rtIs59, increased basal locomotion rates by $38 \%$ (Fig. 2c, $p=0.003$ ).
However, it is unclear whether the increased locomotion of these high copy PLS3 overexpression animals is pertinent to the impact of PLS3 in SMA models. We cannot rule out that a high dosage of PLS3 is detrimental because it has been shown that high levels of the analogous actinbundling protein Sac6 cause lethality in yeast [44]. Therefore, we avoided use of this high copy PLS3 transgene in the rest of this study, unless explicitly noted. a

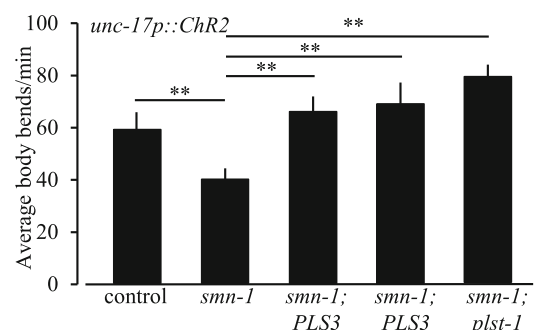

c

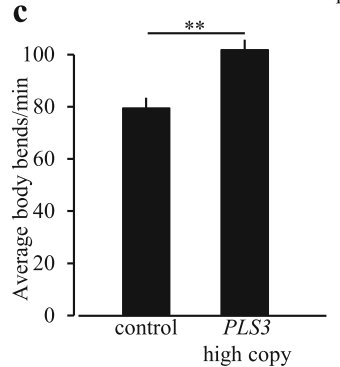

b
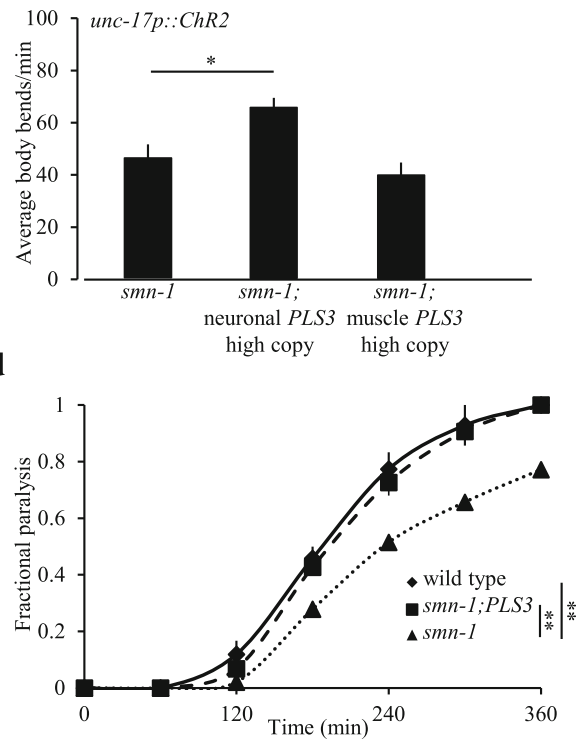

Fig. 2 Neuronal expression of human PLS3 restores normal locomotion in smn-1(cb131) animals. a Locomotion rates of smn-1(cb131) animals decreased after a 20-s blue light stimulation of ChR2 in cholinergic neurons, in the presence of retinal. Introduction of somatic expression of human Plastin 3 (PLS3) restored normal locomotion rates immediately post-exhaustion. Two different PLS3 transgenes were used. Low copy is a homozygous single-copy insertion of [dpy-30p::PLS3], rtSi27 on chromosome II; high copy is an integrated multi-copy transgene incorporating the same transgene as low copy, rtls59. Ubiquitous overexpression of the C. elegans ortholog of PLS3 (plst-1) was equally effective rtEx850. ANOVA $F(2.9,13.5)=3.33, p<0.05$. b Neuronal expression of PLS3 rtEx852 [unc-119p.:PLS3] rescued the post-exhaustion locomotion defects of smn1 (cb131) animals; expression in muscles rtEx851 [myo-3p::PLS3] did not. ANOVA F(1.56, 10.28) $=4.25, p<0.05$. c Overexpression of human PLS3 at high copy levels using rt/s59 increased basal locomotion rates in a wild-type background compared to controls. d smn-1(cb131) mutant animals were resistant to the cholinesterase inhibitor aldicarb, based on slower time to paralysis. Introduction of ubiquitously expressed human PLS3 restored sensitivity to normal levels. Note that C. elegans plst-1 was intact in all genotypes reported here and control animals contained the transgene rtSiz8 [dpy-30p::empty] to account for insertion site and/or multi-copy promoter effects. In all assays, $n \geq 30$ animals per determination, combined from 3 independent trials. Scorers were blinded to the genotypes of animals during the collection and analysis of data for the locomotion and aldicarb sensitivity assays. Student's $t$ test, Mann-Whitney $U$ test $(\mathbf{a}-\mathbf{c})$, or log rank test $(\mathbf{d}):{ }^{*} p<0.05$, ${ }^{* *} p<0.01$. SEM indicated 
Increasing plastin levels ameliorates NMJ and pharyngeal pumping defects caused by decreased smn-1 function smn-1(cb131) animals are resistant to paralysis by the acetylcholinesterase inhibitor, pyridostigmine bromide, suggesting reduced cholinergic NMJ function [35]. We found that diminished SMN-1 function also resulted in decreased sensitivity to aldicarb, another acetylcholinesterase inhibitor. Prolonged exposure to aldicarb gradually induces paralysis, due to acetylcholine accumulation in the synaptic cleft. smn-1(cb131) animals paralyze more slowly on aldicarb than control animals (Fig. 2d, $\log$ rank $p=0.009$ ). Introduction of the human PLS3 multi-copy transgene into smn1(cb131) animals restored normal aldicarb sensitivity (Fig. 2d, $p=0.009$ versus transgenic control lacking PLS3). These results indicate that ubiquitous PLS3 expression improves cholinergic NMJ function when SMN-1 is impaired.

Various studies show that the effects of PLS3 may depend on the levels of SMN present. In models where SMN levels are substantially decreased, increased PLS3 insufficiently suppresses neuromuscular defects $[15,16]$. However, to test if the beneficial impact of PLS3 is not limited to smn-1(cb131) animals, we also tested smn-1(ok355) severe loss of function animals, which contain even lower levels of SMN-1 activity compared to smn-1(cb131). smn-1(ok355) animals have decreased pharyngeal pumping rates, a behavior frequently used to assess neuromuscular function. C. elegans feed on bacteria using a dedicated set of muscles and neurons in the pharynx. Pharyngeal pumping rates average roughly 250 pumps per minute in the presence of food; late larval stage smn-1(ok355) animals have dramatically reduced pharyngeal pumping rates [31, 39]. In control animals with a wild-type $s m n-1$ gene, ubiquitous expression of human PLS3 in C. elegans slightly decreased pumping rates (single-copy transgene, $r$ Si27, $-11 \%$ versus wild type, Additional file 1: Fig. S1, $p=0.002$ ). However, the introduction of the single-copy PLS3 transgene into smn-1(ok355) animals increased pharyngeal pumping rates by $50 \%$ (Additional file 1: Fig. S1, versus ok355 $p=0.011$ ). Although pumping rates were not restored to wild-type levels, we conclude that in severe loss of function smn-1 animals, increased PLS3 partially ameliorates neuromuscular defects. Using two different alleles and testing multiple defects, an overexpression of PLS3 proves beneficial when SMN levels are decreased in C. elegans. This concurs with the partial amelioration observed in particular type IIIb SMA patients that carry two SMN2 copies and have elevated levels of PLS3 [12]. Taken together, our results demonstrate that plastin is a conserved genetic modifier of neuromuscular function.
Identification of sym-2 as a genetic modifier of both PLS3 and smn-1

PLS3, SMN, and actin can be co-precipitated from HEK293T cells and murine spinal cord, but SMN does not directly interact with PLS3. The identity of other proteins in this complex is unknown [12]. Delineating other proteins in the PLS3/SMN complex would lead to a better understanding of SMA and the protective mechanism engaged by increased PLS3 levels. To identify these other proteins, we undertook a small, targeted screen starting with results from a previous large-scale proteomic study undertaken in Drosophila S2 cells [40]. From those results, we assembled a list of proteins that pulled down either with Drosophila SMN or with Drosophila Fimbrin, the PLS3 ortholog. We prioritized proteins found in both lists, based on frequency of pull-down; protein similarity between $D$. melanogaster, $H$. sapiens, and C. elegans; and the availability of C. elegans loss of function $(l f)$ alleles. From this, eleven C. elegans candidate genes were targeted for functional analysis (Fig. 3a). We hypothesized that proteins in the same protein complex as SMN and PLS3 might show functional interactions, and we interrogated these putative interactions by testing if C. elegans candidate gene loss of function suppressed PLS3and SMN-associated defects. High copy PLS3 expression results in aberrantly high locomotion levels in liquid (Figs. $2 \mathrm{c}$ and $3 \mathrm{~b}$ ). We determined if candidate gene loss of function alleles could suppress locomotion changes caused by increased PLS3.We also determined if candidate gene loss of function could suppress the defect in smn-1(cb131) recovery from ChR2-induced exhaustion. Only sym-2 loss of function ameliorated defects in these assays (Fig. 3b, $p=$ 0.007 , and $\mathrm{c}, p=0.006$ ). RNAi knockdown of sym-2 also suppressed smn-1(cb131) ChR2-induced exhaustion recovery defects (Additional file 1: Fig. S2, $p=$ 0.02, smn-1(cb131) control versus knockdown of sym-2), but knockdown of genes encoding two related C. elegans proteins had no impact (hrpf-1 and hrpf-2, Additional file 1: Fig. S2). Glorund was identified from the proteomic Drosophila screen, and BLAST results list the orthologs sym-2, hrpf-1, and hrpf-2 in C. elegans. BLAST results also show the human orthologs to glorund as hNRNP F, H1, H2, GRSF1, and ESRP 1 and 2. Based on predicted functional domains and expression patterns, the human proteins most closely related to glorund and SYM-2 are hnRNP F, hnRNP H1, and hnRNP H2 [46]. Members of this heterogeneous nuclear ribonucleoprotein family have roles in pre-mRNA processing [42, 47, 48]. Given the results above, we chose to focus on sym-2 for the remainder of this study. 


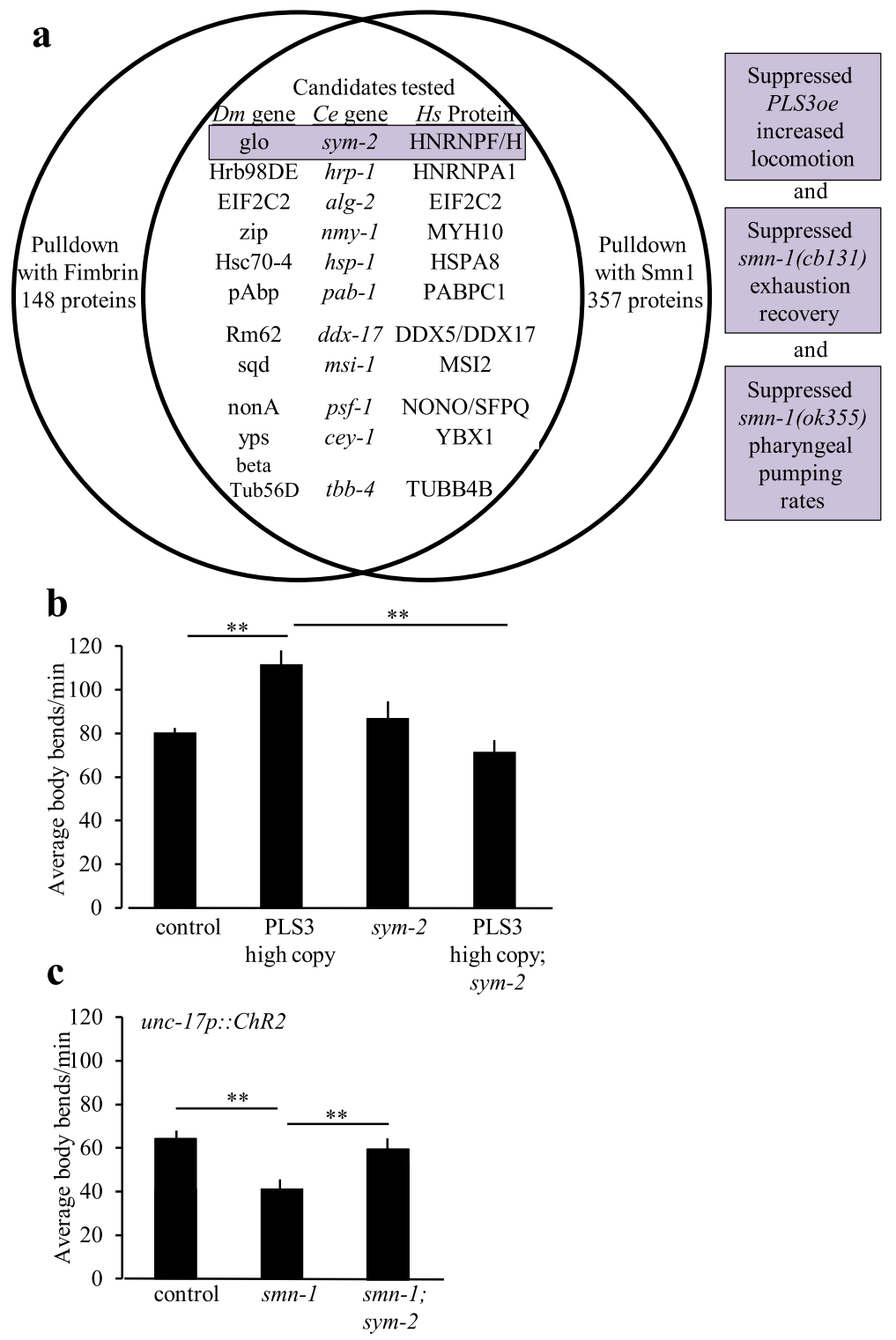

Fig. 3 Identification of sym-2 as a suppressor of PLS3oe and smn-1 locomotion defects. a To find proteins in a putative SMN/PLS3 complex, we focused on candidates that independently pulled down in previous work [45] with both Drosophila Fimbrin and Drosophila Smn (left and right circles of Venn diagram, details in the "Methods" section). Candidates that had a corresponding C. elegans loss of function allele available in 2011 are listed and were examined. Only sym-2 loss of function suppressed PLS3oe and smn-1 defects in all three assays (right) used to screen these candidates. b Overexpression of human PLS3 increased basal locomotion rates compared to control animals. PLS3 was overexpressed from rtls59, an integrated multi-copy transgene ubiquitously driving expression using the $d p y-30$ promoter. Decreased function of the C. elegans hnRNP F/H ortholog, sym-2, did not change locomotion rates in a wild-type background, but sym-2 perturbation suppressed increased locomotion in PLS3oe animals. ANOVA $F(7962,663)=175.96, p<0.05$. c sym-2 perturbation suppressed locomotion defects in smn-1(cb131) animals immediately following ChR2 exhaustion. All animals in c carried ox/s364[unc-17p::ChR2]; ANOVA $F(0.84,0.55)=17.51, p<0.05$. In all assays, $n \geq 30$ animals per determination were used, combined from 3 independent trials. Scorers were blinded to the genotype of animals during the collection of data and data analysis. Student's $t$ test: ${ }^{* *} p<0.01$. SEM indicated

sym-2(mn617) is a Y163N missense allele that likely decreases SYM-2 function [46]. As described above, introduction of sym-2(mn617) into the PLS3 overexpression background returned locomotion rates to normal (Fig. 3b, $p=0.007$ ). And, introduction of sym2(mn617) ameliorated smn-1(cb131) post-exhaustion locomotion defects in double mutant animals (Fig. 3c smn-1(cb131); sym-2(mn617) versus smn-1(cb131), $p=$ 0.006). Taken together, these results demonstrate that decreased sym-2 function suppresses defects caused by decreased smn-1 activity or increased PLS3 function. 


\section{PLS3 colocalizes with SMN and SYM-2 orthologs in vertebrate neurons}

SMN-1, PLS3, and SYM-2 may act in a common functional pathway, but $C$. elegans genetic studies cannot determine if these proteins act in a conserved protein complex in mammalian neurons. Previously, SMN protein was found in RNP granules with various hnRNPs in neuronal processes, but not with hnRNP F/H orthologs [48-50]. While the majority of studies focus on the nuclear role in splicing of hnRNP $\mathrm{F} / \mathrm{H}$, one cannot exclude a possible role outside the nucleus $[51,52]$. Therefore, a series of immunohistochemical assays were done to look for an association of hnRNP F/H with PLS3 and SMN that might be pertinent in cells and occur outside the nucleus.

First, we examined localization of PLS3, SMN, hnRNP $\mathrm{F}$, and hnRNP $\mathrm{H} 1 / 2$ in murine fibroblasts. Primary cultures were generated from mice overexpressing a V5tagged PLS3 [16]. As expected, all four proteins were detected in fibroblast nuclei using immunohistochemistry. Interestingly, all four proteins are also present within cytoplasmic filopodia, suggesting they function in the same cellular compartment (Fig. 4a-d).

Next, we examined localization of hnRNP F, hnRNP $\mathrm{H} 1 / 2$, PLS3, and SMN in the processes of cultured mouse motor neurons. Embryonic stem cells were used to generate neuronal cultures from mice expressing motor neuron GFP (under the control of the Hb9 promoter). After 13 days of growth in culture, endogenous SMN, PLS3, hnRNP F, and hnRNP H1/2 proteins were detected within neuronal processes using immunohistochemistry. Each protein was distributed within motor neuron processes. To quantitatively assess protein localization within these structures, we used semiautomated image analysis software (Columbus, Perkin Elmer). By defining protein presence based on size, this analysis showed that SMN independently localized to the same region with all three proteins of interest in these structures (Fig. 4e-g), allowing us to conclude that hnRNP F and H1/2 are found in fibroblasts and in neuronal processes of motor neurons and that these proteins frequently localize to the same region with SMN. Collectively, these cellular data suggest that an association may happen between SMN, PLS3, hnRNP F, and H1/2 in vivo.

\section{SMN, PLS3, and SYM-2 orthologs co-exist in vertebrate protein complexes}

The localization analysis suggests that these proteins might be found in a similar cellular compartment and therefore could be in a complex together, a question we addressed using co-immunoprecipitation studies. HEK293T cells were transfected with tagged versions of all four proteins. Immunoprecipitation of GFP-tagged
hnRNP $\mathrm{F}$ or hnRNP $\mathrm{H} 1 / 2$ revealed that these proteins are associated with both SMN and PLS3 (Fig. 5a). The interaction was not RNA-dependent, as associations were maintained after RNase treatment (Additional file 1: Fig. S3). These results are consistent with the localization analysis that show an interaction between the proteins.

Co-immunoprecipitation from mouse brain lysates confirmed an endogenous interaction between SMN and PLS3, hnRNP F, or hnRNP H1/2 (Fig. 5b bracket) in neuronal tissues. Reciprocally, endogenous PLS3 coimmunoprecipitated with SMN, hnRNP F, and hnRNP $\mathrm{H} 1 / 2$ (Fig. 5c). We conclude that SMN and PLS3 are in protein complexes that also contain hnRNP $\mathrm{F}$ and/or hnRNP $\mathrm{H} 1 / 2$ in the mouse brain. However, from this evidence alone, one cannot conclude that hnRNP $\mathrm{F}$ or hnRNP $\mathrm{H} 1 / 2$ are in a complex simultaneously containing both SMN and PLS3.

To test if these proteins are found in a complex together, we undertook size fractionation of whole cell lysates using FPLC columns and HEK293T cells expressing tagged versions of PLS3 and hnRNP F. In complexes of less than $669 \mathrm{kDa}$ and greater than $158 \mathrm{kDa}$, four proteins-PLS3, SMN, actin, and hnRNP F-were found (Fig. 5d). And, in the smaller complexes within this range, hnRNP H1/2 also were found. Note that most of the PLS3, actin, and hnRNP F/H1/2 proteins are found in even smaller complexes, consistent with previously described interactions of these proteins. Taken together, size fractionation, co-immunoprecipitation, and localization results suggest that a rare previously unidentified complex may exist that contains actin, SMN, and PLS3 as well as hnRNP F and/or hnRNP H1/2.

\section{SYM-2 loss rescues smn-1 endocytosis defects}

Fluid-phase endocytosis is perturbed in C. elegans with decreased smn-1 function [8]. For mechanistic insight in how sym-2 loss suppresses smn-1 defects, we used a classical C. elegans assay which assesses endocytosis in coelomocyte cells. Six coelomocyte cells are found in the body cavity (pseudocoelom) of adult C. elegans. These cells play a critical role in the active removal of proteins by endocytosis $[53,54]$. When muscle cells in transgenic animals secrete soluble GFP into the pseudocoelom, GFP is taken up by coelomocytes through fluid-phase endocytosis and is sent for degradation. This assay has been used extensively to characterize endocytic pathways [55]. RNAi knockdown of smn-1 in coelomocytes impairs GFP uptake and GFP clearance from the body cavity, consistent with endocytic pathway defects [8]. We found that either PLS3 overexpression or coelomocytespecific RNAi knockdown of sym-2 ameliorated endocytic defects in smn-1(RNAi) animals. Overexpression of PLS3 reduced the number of animals with GFP 


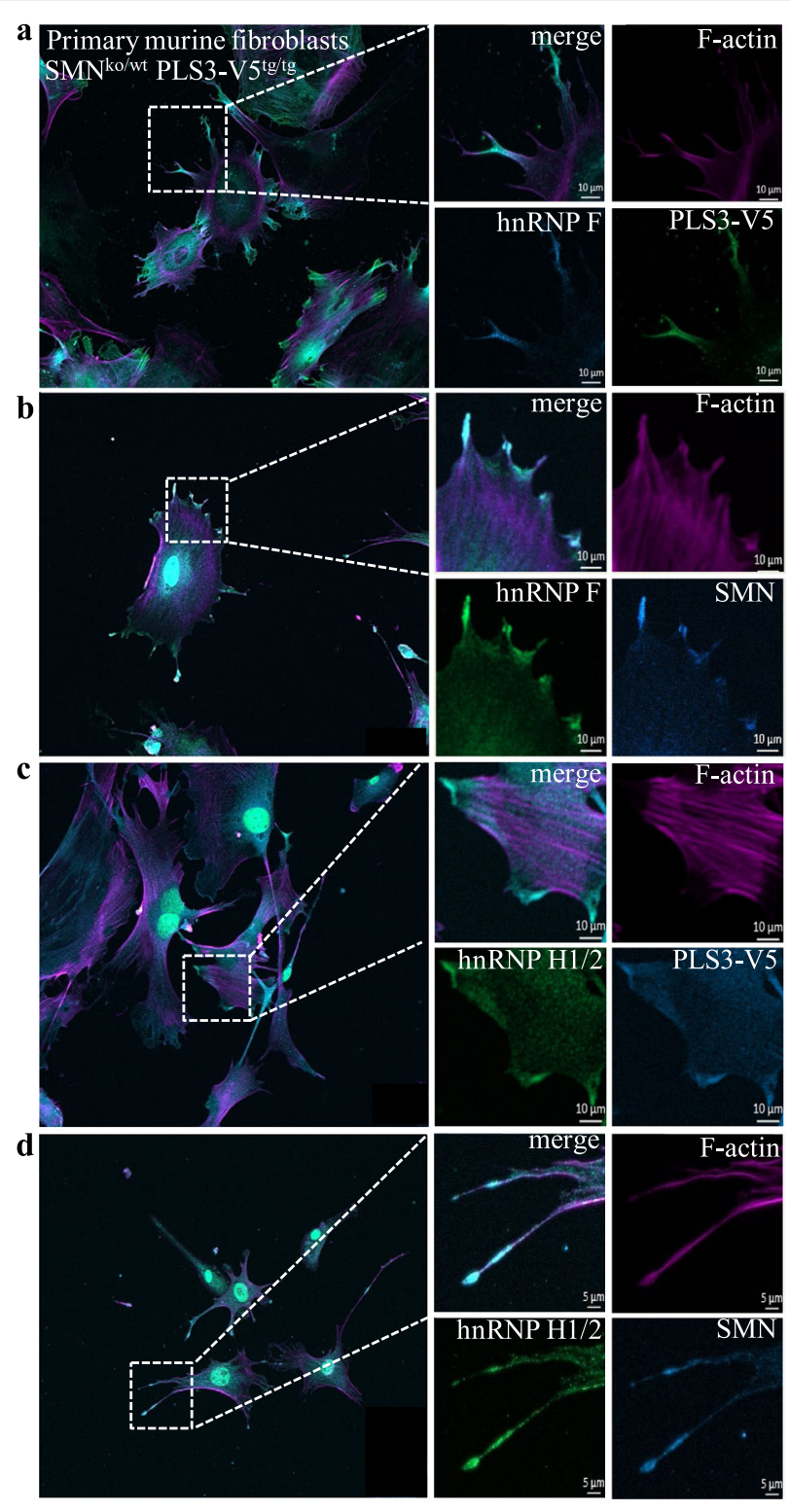

Mouse primary motor neuron processes
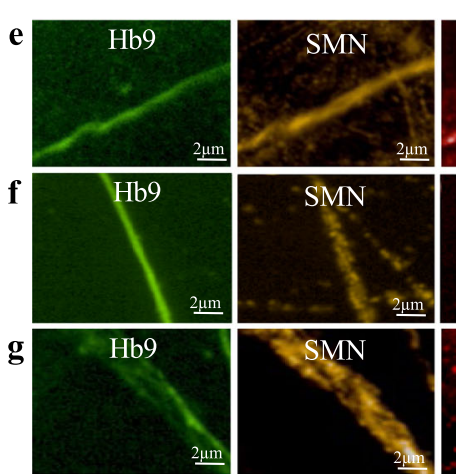

Fractional puncta overlap

SMN/PLS3

0.41

SMN/hnRNP F

0.54 
(See figure on previous page.)

Fig. 4 hnRNPF and hnRNPH1/2 localize with SMN and PLS3 in fibroblasts and motor neuron processes. Murine embryonic fibroblasts' (MEFs) cell cultures were derived from Smn ${ }^{+-}$; PLS3V5 ${ }^{\text {tg/tg }}$ embryos and stained with V5 for PLS3 transgene; hnRNP-H1/2, hnRNP-F, and F-actin with phalloidin. a PLS3V5 localized to the same region as hnRNP F. b hnRNP F similarly localized with SMN. c hnRNP H1/2 localized to the same region with tagged PLS3. $\mathbf{d}$ hnRNP H1/2 localized to the same region with SMN. e SMN localized with PLS3 also in mouse spinal motor neurons processes in primary culture. Motor neurons were derived from GFP-Hb9-expressing embryos, which labels motor neurons. Endogenous protein was detected using immunohistochemistry. Quantitative analysis of cellular localization using confocal images of processes reports the percentage of localization for SMN fluorescence within processes. Protein fluorescence criterion was manually set using controls within the COLOMBUS software (Perkin Elmer), see the "Methods" section for details. $\mathbf{f}$ Localization of SMN with hnRNP F was seen using the same methods as described in $\mathbf{e} . \mathbf{g}$ Localization of SMN with hnRNP H1/2 was seen using the same methods as described in e. Protein localization assays were done as three independent trials where each sample in each trial was a merge of images from multiple wells on a 96-well plate. Images were taken using an automated program, and then, slices were chosen incorporating planes with the nuclei

accumulation in the pseudocoelom (Fig. 6, $p=0.039$ ), consistent with the previously demonstrated role of PLS3 in endocytic pathways [9]. Moreover, RNAi knockdown of $s y m-2$ dramatically improved GFP clearance from the pseudocoelom of $s m n-1$ (RNAi) mutant animals
(Fig. 6, $p=0.1 \times 10^{-5}$ ). These results demonstrate that the endocytic pathway defects observed when smn-1 levels drop are counteracted by knockdown of $s y m-2$ in a cell autonomous manner. Combined with previous work, these results suggest that SMN, PLS3, and hnRNP
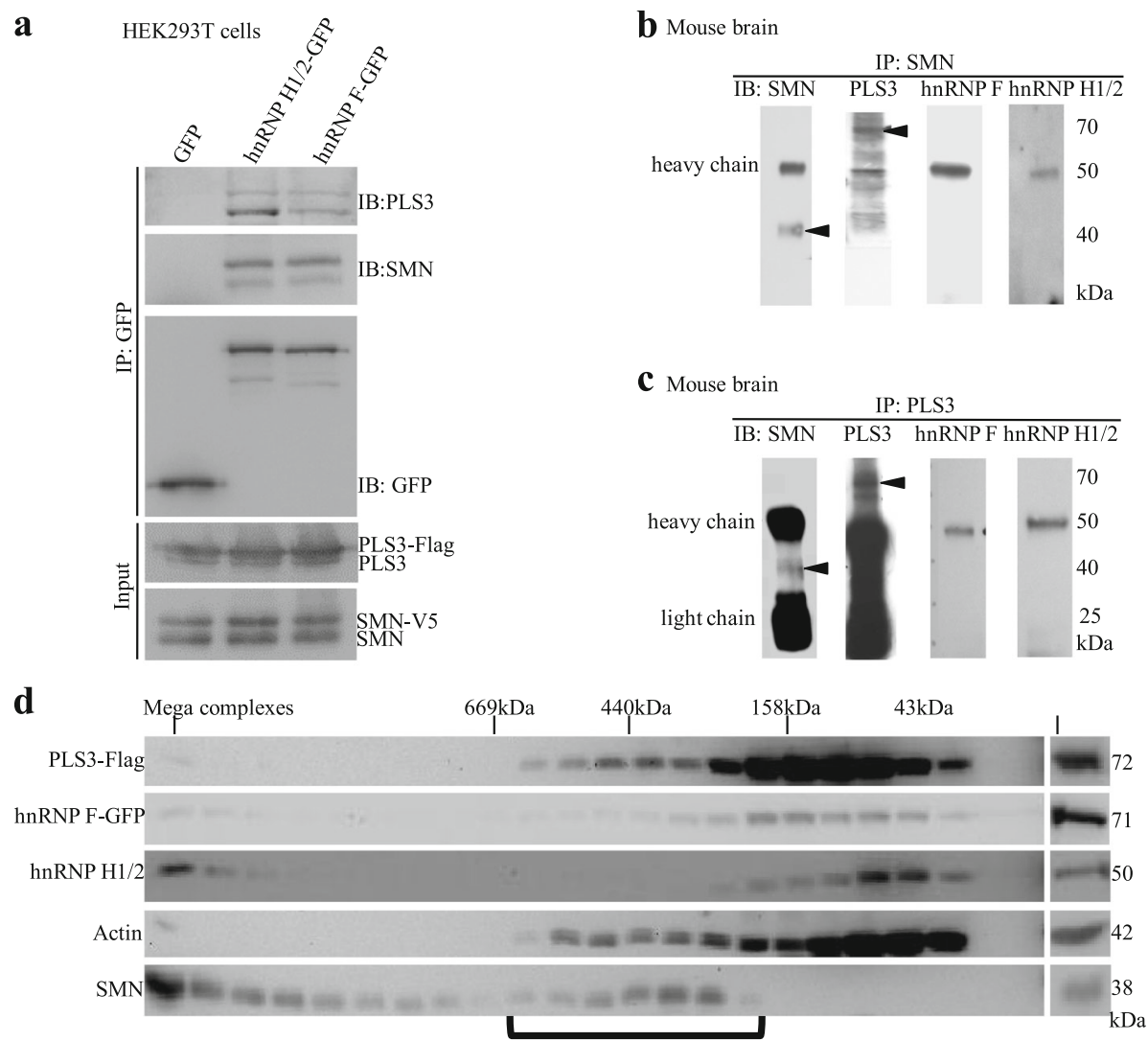

Fig. 5 SMN, PLS3, hnRNP F, and hnRNP H1/2 associate in a large complex. a SMN and PLS3 independently associated with hnRNP H1/2 based on co-immunoprecipitation from HEK293T cells transfected with tagged proteins. b Endogenous PLS3, hnRNP F, and hnRNP H 1/2 associated with SMN in mouse brain extracts, based on immunoprecipitation with antisera against endogenous SMN protein. Arrowheads indicate the band of interest in blots with multiple bands. Heavy and light chain bands are labeled when present. c Endogenous SMN, hnRNP F, and hnRNP H1/2 associated with endogenous PLS3 using the same approach outlined in $\mathbf{b}$. Arrowheads indicate the band of interest in blots with multiple bands. Heavy and light chain bands are labeled when present. IP on endogenous mouse brain extract was done from 3 independent brain extractions and preparations. $\mathbf{d}$ Size fractionation studies of whole cell lysates from HEK293T cells showed that actin, SMN, PLS3, and hnRNP F and/or H1/2 proteins co-fractionate in complexes of the indicated size (bracket), although the bulk of hnRNP F/H1/2 protein is in other smaller complexes. HEK293T cells were transfected with PLS3-Flag, and hnRNP F-GFP, endogenous hnRNP H1/2, actin, and SMN were detected using antisera 

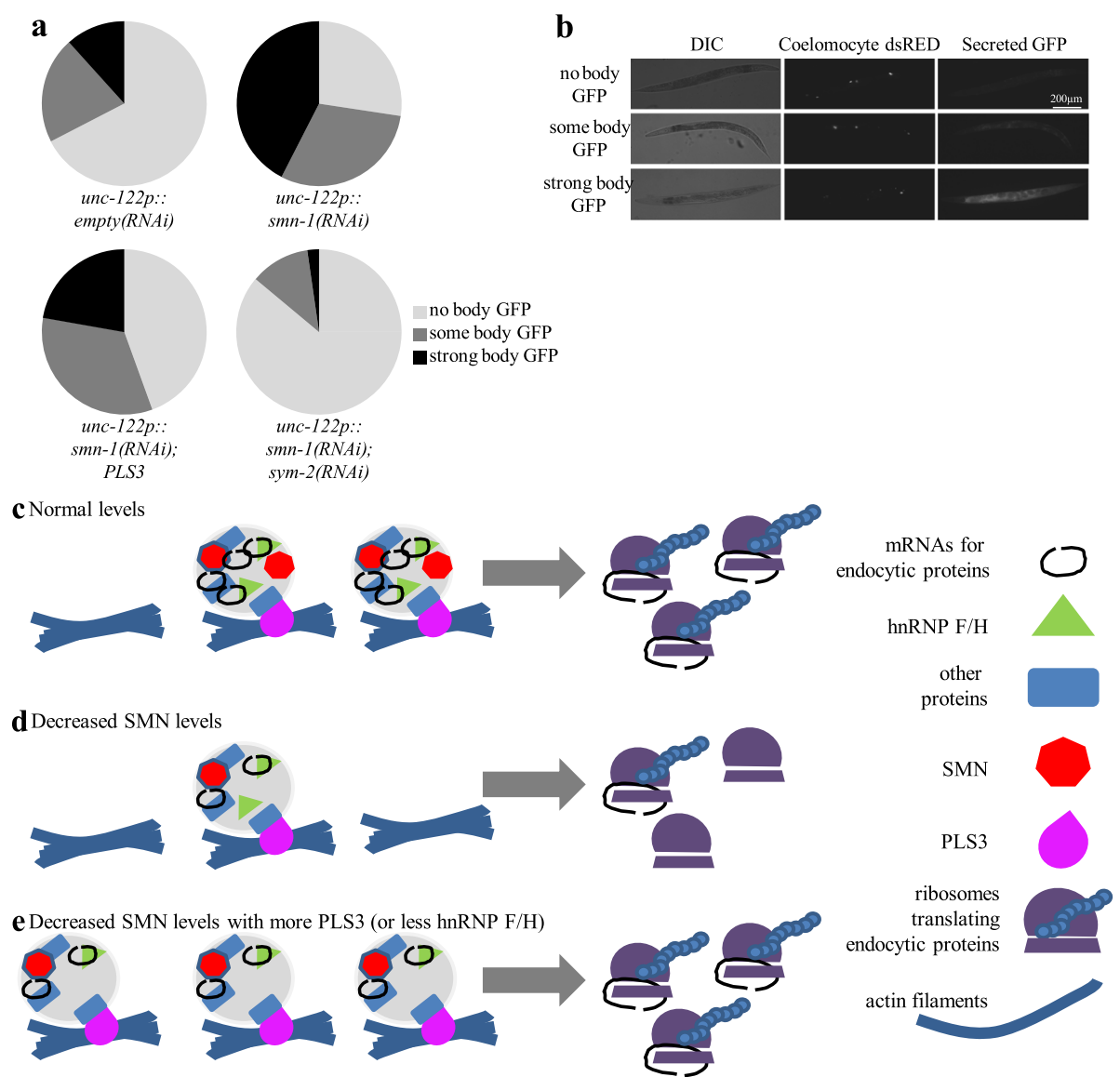

Fig. 6 PLS3 overexpression and sym-2(RNAi) suppress endocytic defects caused by smn-1(RNAi) knockdown and summary. a Pie charts illustrating the percentage of animals with the indicated accumulation of GFP in the body cavity. Soluble GFP is secreted from the muscle cells into the body cavity. Coelomocyte-specific knockdown of smn-1 leads to GFP accumulation in the body cavity due to endocytic pathway defects. Either coelomocyte-specific expression of PLS3 or coelomocyte-specific knockdown of sym-2 suppressed GFP accumulation, consistent with cellautonomous action of these proteins in coelomocytes. $n>40$ animals pooled from multiple extrachromosomal array lines, combined from 3 independent trials. Scorers were blinded to the genotype of animals for each trial. Statistics calculated using chi-square. b Representative images for "no body GFP," "some body GFP," and "strong body GFP." c Based on results presented here and previous work, a summary of functional genetic interactions is shown. c Normal levels of SMN protein facilitate appropriate transport and translation of "endocytic mRNAs," which encode proteins involved in endocytosis. As an actin-bundling protein, PLS3 may contribute to transport or localization of RNP granules (gray circles) that contain "endocytic mRNAs," as well as SMN, hnRNPF/H, and other proteins. d When SMN levels drop, the number, trafficking, or function of RNP granules containing "endocytic mRNAs" is perturbed, resulting in fewer endocytic proteins and endocytic defects. e The deleterious

consequences of decreased SMN levels can be ameliorated by increasing PLS3 levels or by decreasing hnRNP F/H levels. The illustration of SMN and hnRNP F/H in an RNP tethered by PLS3 is speculative, but increasing PLS3 or decreasing hnRNPF/H ortholog activity ameliorates both functional defects and endocytic defects in the C. elegans model

$\mathrm{F} / \mathrm{H}$ act in a protein complex, pertinent to SMA, whose function is required for normal function of endocytic pathways.

\section{PLS3 expression suppresses defects in a C. elegans ALS model}

Increasing PLS3 levels accelerates neurite outgrowth in vertebrate motor neurons, regardless of SMN status, suggesting that increased PLS3 might impact general function and survival of motor neurons [12]. With the notion that common mechanisms may underlie neurodegenerative diseases, increased PLS3 expression may modify defects associated with other neurodegenerative diseases besides SMA [16, 20, 56]. Based on this hypothesis, we determined if increasing PLS3 levels would modify defects in an established C. elegans model of ALS [30].

In the previously characterized C. elegans ALS model, wild-type human SOD1 (SOD1-WT) or mutant human SOD1 carrying the patient amino acid change G85R (SOD1-G85R) are expressed at high levels using a panneuronal C. elegans promoter (from the snb-1 gene). Mutant animals at young adult stage showed decreased locomotion in liquid, decreased pharyngeal pumping 
rates, and resistance to aldicarb, compared to their corresponding control animals (Additional file 1: Fig. S4, $p=6 \times 10^{-17}, \quad p=1.1 \times 10^{-8}, \quad p=6.6 \times 10^{-15}, \quad p=3.7 \times$ $10^{-10}, p=4.7 \times 10^{-4}, p=1.41 \times 10^{-8}$ ) [30]. We introduced the single-copy PLS3 transgene rtSi27 into both SOD1WT and SOD1-G85R to determine if these defects would be suppressed.

Expression of PLS3 in SOD1-G85R animals proved to be beneficial. Locomotion rates increased $61 \%$ compared to SOD1-G85R animals lacking PLS3 (Additional file 1: Fig. S4a, $p=3 \times 10^{-6}$ ). Pharyngeal pumping defects were similarly ameliorated; PLS3 expression increased pumping rates by $35 \%$ in SOD1-G85R animals, restoring pumping to normal levels (versus SOD1-G85R without PLS3, Additional file 1: Fig. S4b, $p=4.1 \times 10^{-8}$ ). Sensitivity to paralysis by aldicarb was also restored in SOD1G85R animals expressing additional PLS3, compared to SOD1-G85R animals without PLS3 (Additional file 1: Fig. S4c, $p=0.002$ ). Overexpression of PLS3 does not indiscriminately increase locomotion or pumping, nor does it always change aldicarb sensitivity. SOD1-WT animals expressing PLS3 showed decreased locomotion rates, compared to SOD1-WT animals without PLS3 (Additional file 1: Fig. S4a, $p=1.7 \times 10^{-5}$ ). PLS3 had no impact on pharyngeal pumping rates or aldicarb sensitivity in SOD1-WT animals (Additional file 1: Fig. S4b and 4c). Overexpression of SOD1-WT results in motor dysfunction, specifically in locomotion assays [57]. While we observed this defect as well, it is unclear why overexpression of PLS3 is detrimental to SOD1-WT animals when assessing locomotion but not for other behaviors. However, due to the consistent beneficial effects in mutant SOD1-G85R animals, we conclude that increased plastin levels can suppress defects in a C. elegans model of ALS.

To determine if PLS3 is beneficial outside of neurodegenerative disease models, we examined the impact of overexpression on animals with decreased locomotion due to synaptic defects. The unc-25 gene encodes the $C$. elegans ortholog of mammalian GAD1 and is required for $y$-aminobutyric acid (GABA) synthesis [58]. The behavioral defects of these animals include a $65 \%$ decrease in locomotion rates in liquid (Additional file 1: Fig. S5, $p=5 \times 10^{-25}$ unc-25(e156) versus wild type). PLS3 expression did not increase locomotion rates $(p=0.576$ versus $u n c-25)$. The $u n c-13$ and $u n c-57$ genes encode $C$. elegans orthologs of mammalian $\mathrm{UNC} 13$ proteins and Endophilin A protein, which are critical for presynaptic vesicular release and endocytosis, respectively. These mutant animals move in an uncoordinated manner compared to wild-type animals. PLS3 expression did not increase locomotion of animals lacking unc-13 function, but did increase locomotion in animals lacking unc-57 function (Additional file 1: Fig. S5, $p=0.02 u n c-57$ versus unc-57 with PLS3oe). Taken together, these results indicate that PLS3 overexpression is not generically beneficial and ameliorating endocytic pathways may improve function in C. elegans models of motor neuron disease.

\section{SYM-2 loss also improves locomotion in a C. elegans ALS model}

Considering that PLS3 overexpression suppressed defects in a C. elegans disease model of ALS and the relationship between PLS3 and SYM-2 described above, it seemed plausible that $s y m-2$ might also be a modifier in an ALS model. We assessed the impact of sym-2 on locomotion in the C. elegans SOD1 overexpression model. Using RNAi to knockdown sym-2 levels, SOD1-G85R animals had increased locomotion rates with sym-2(RNAi) compared to empty vector RNAi feeding (Additional file 1: Fig. S4d, versus control (RNAi), $p=3.7 \times 10^{-8}$ ). These results suggest that, reminiscent of PLS3, the beneficial impact of hnRNP $\mathrm{F} / \mathrm{H}$ loss and endocytic pathway changes may extend to other motor neuron diseases.

\section{Discussion}

Elucidating how the pathology and symptoms of motor neuron diseases can be suppressed should yield important insights into neurodegenerative mechanisms. Here, we focused on understanding functional interactions between PLS3 and SMN, and on how their interaction provides insight into pathways pertinent to disease. We confirm that increasing PLS3 suppresses locomotion defects and endocytic pathway defects that are caused by decreased SMN levels. In addition, we identify hnRNP $\mathrm{F} / \mathrm{H}$ as a component of protein complexes containing SMN and PLS3. We demonstrate that decreasing levels of the C. elegans hnRNP F/H ortholog SYM-2 also suppresses both locomotion and endocytic pathway defects caused by diminished smn-1 function. Additionally, we find that the beneficial impact of increased PLS3 or decreased SYM-2 expression is not limited to SMA; defects in a C. elegans model of ALS are also suppressed. Finally, we show that interactions identified in C. elegans are likely conserved, as PLS3, SMN, hnRNP F, and/or hnRNP H1/2 are found together in mammalian neuronal tissues and motor neuron processes. Combined, these results suggest that SMN and PLS3 act to ameliorate SMA defects via protein complexes that contain hnRNP $\mathrm{F}$ and/or H1/2 that is pertinent to endocytic pathways.

Using mouse fibroblast cells and primary motor neurons, we demonstrated that PLS3, SMN, hnRNP F, and hnRNP H1/2 have an association and localize to the same regions in vitro. Biochemical analysis confirmed these findings in HEK293T cells and mouse brain extracts. Additionally, fractionation studies from mouse 
neuronal tissues and/or HEK293T cells suggested that PLS3, SMN, and hnRNP F and/or H1/2 proteins can be found in protein complexes. Given the biochemical interactions identified in this study, the genetic interactions observed between SMN-1, PLS3, and SYM-2 likely also exist between the vertebrate orthologs SMN, PLS3, and hnRNP $F / H 1 / H 2$. Discovering how hnRNP $F / H$ family proteins interact with SMN and PLS3 will yield insights into aspects of their function critical in disease. hnRNPs are a diverse group of RNA binding proteins originally named for their ability to bind hnRNAs (heterogeneous nuclear ribonucleic acids) [47]. hnRNP proteins have diverse roles and are most commonly involved in pre-mRNA processing, including mRNA export, localization, translation, and stability [42]. While studies have identified that altering hnRNP F/H expression affects proliferation of cancer cells, virtually nothing is known about their role or what altering hnRNP $\mathrm{F} / \mathrm{H}$ expression levels would do in a neurological context. Here, we find that loss of SYM-2 suppresses endocytic pathway defects in animals with decreased SMN-1 activity, identifying a new role for this specific hnRNP subfamily. Understanding the function of hnRNPs beyond their canonical role and identifying how they impact endocytic pathways may lead to a better understanding of endocytosis and SMA.

In C. elegans, mouse, and zebrafish models of SMA, endocytic defects are seen at the NMJ [8-10]. In Huntington's and ALS models, clathrin-mediated endocytosis is perturbed, potentially by protein aggregates that compete with clathrin for chaperone proteins [59]. Furthermore, mutations in CHMP2B, part of a complex involved in endosome-lysosome fusion, cause frontotemporal dementia [60]. Given these commonalities, we speculate that perturbation of endocytic pathways may be a critical step in neurodegenerative disease pathology. Using genetic and functional studies, we showed increased PLS3 expression or decreased SYM-2 expression ameliorated endocytic pathway defects in a model of SMA. This data reinforces that endocytic pathway defects are critical in motor neuron disease and further highlights the importance of understanding why these hnRNPs are important in endocytosis.

Why does increasing PLS3 expression or decreasing SYM-2 expression ameliorate behavioral defects in models of SMA or ALS? Our results demonstrate that these proteins colocalize with SMN, in motor neuron processes. But how does this connect motor neuron disease to endocytic pathways? Previous work has shown that increased PLS3 does not increase overall SMN protein levels $[12,15]$. However, increased PLS3 may lead to stabilization or transport of specific RNP complexes that are disrupted when SMN levels decrease. Previous work also demonstrated that a minimal amount of SMN is required for PLS3 to ameliorate symptoms, consistent with the more dramatic impact of PLS3 overexpression on smn-1(cb131) partial loss of function versus smn1 (ok355) complete loss of function animals. Previous studies have also analyzed animals with decreased plastin levels. In smn mutant zebrafish, PLS3 protein levels were found to correspondingly decrease when SMN levels decreased. However, this correlation was not repeated when profilin II levels were looked at, thus suggesting the interaction with PLS3 is unique [38].

Using the results presented herein along with previously published work, we suggest a model in which the balanced and coordinated action of SMN, PLS3, and hnRNPF/H proteins promotes the optimal transport and/or function of mRNAs and their encoded proteins, and further that these mRNAs and proteins are essential for normal endocytic function (Fig. 6). We suggest that decreased levels of SMN negatively impact mRNAs required for endocytosis and that appropriate increased expression of PLS3 or decreased expression of hnRNP $\mathrm{F} / \mathrm{H}$ levels can counteract these negative impacts. As summarized in Fig. 6, decreased SMN protein levels may lead to decreased function or transport in axons of RNP granules containing hnRNP $\mathrm{F} / \mathrm{H}$. However, increasing PLS3 leads to improved RNP granule transport or function, despite diminished SMN levels. Decreasing hnRNP $\mathrm{F} / \mathrm{H}$ may improve the translation and/or function of endocytic proteins encoded by mRNAs within these granules. In either case, increasing PLS3 or decreasing hnRNP F/H improves endocytic function, which partially ameliorates the deleterious consequences of diminished SMN function.

\section{Conclusions}

Genetic studies are important in understanding disease pathology and can yield novel and unexpected insights into disease mechanisms. This is exemplified by the identification of PLS3 and NCALD as modifiers of SMA in patient families and the identification of hnRNP $\mathrm{F} / \mathrm{H}$ as a genetic modifier herein. Based on the level of conservation observed, it seems likely that decreasing hnRNP $\mathrm{F} / \mathrm{H}$ protein function would also be beneficial in vertebrate SMA models, and potentially in ALS models as well. The identification and functional interaction identified between SMN, PLS3, and hnRNP F/H provide insight into the mechanisms that lead to perturbations in motor neuron disease.

\section{Methods}

C. elegans strains were maintained at $20^{\circ} \mathrm{C}$ under standard conditions (Additional file 1: Table 1) [61]. All animals in experiments with smn-1(ok355) were first generation progeny of heterozygous for the $h T 2$ balancer to preserve a uniform genetic background. RNAi studies 
were undertaken in a sensitized background containing the transgene, uIs72, that expresses the SID-1 doublestranded RNA channel in neurons [62]. RNAi clones were verified by sequencing and aligned for specificity to the gene of interest. Only one RNAi clone was tested for each gene of interest; however, in the case of smn-1 and sym-2, loss of function alleles showed the same phenotype in behavioral testing. For behavioral assays with transgenic animals, at least 3 integration lines were tested to confirm results and rule out behavioral phenotypes due to the integration site. All integrated transgenes were backcrossed to wild type at least three times before final strain construction. The transgenic strains rtSi27 and rtSi28 were generated by Mos-mediated single-copy insertion [63]. The $d p y$-30 promoter was cloned into pPD49.26 from the C. elegans expression vector $\mathrm{pS} 235$, containing the $d p y-30$ promoter via $\mathrm{SphI}$ and NheI sites. Human PLS3 CDS (ATG to TAA) was amplified from pcDNA3.1_PLS3.V5-His6 TOPO vector [9] using primers 5'-GAACGCTAGCATGGATGA GATGGCTACCAC-3' and 5'-CAGGGGAATGAAGA GAGTGTAACCCGGGGTTC-3' and cloned behind the $d p y$-30 promoter creating $d p y$-30p::PLS3. Either the promoter alone or the promoter with the PLS3 coding sequence was cloned into a modified pCFJ66 via SphI and NheI or SphI and XmaI sites, respectively. The final constructs pHA\#606 dpy-30p::unc-54UTR, unc-119(+) and pHA\#607 dpy-30p::PLS3::unc-54UTR, unc-119(+) were injected at $50 \mathrm{ng} / \mu \mathrm{l}$ with the standard MosSCI cocktail and injected into EG4322 (unc-119(ed3)III;cxTi10816 IV) animals. Single-copy insertion events were identified based on rescue of $u n c-119$ in non-fluorescent animals and confirmed by PCR genotyping. Transgenic strains for neuronal and muscle-specific rescue were generated by cloning the $u n c-119$ or myo-3 promoters using SphI and NheI sites into pHA\#606 and pHA\#607, in place of the $d p y$-30 promoter. Both single-copy and multi-copy transgenes were generated by injection of $50 \mathrm{ng} / \mu \mathrm{l}$ of targeting plasmid into EG4322 (unc-119(ed3)III; cxTi10816 IV) animals. Multi-copy insertion events were identified based on the rescue of unc-119 in animals expressing fluorescent markers. Ceplst-1 overexpression animals were created by amplifying plst-1 from the yk1465f08 clone using primers 5'-GGTACCGGTT TAATTACCCAAGTTTG-3' and 5'-GAGCTCCAGA AAAACCGAAAAAATCC-3'. The cDNA was placed behind the $d p y-30 p$ in pPD49.26 using KpnI and SacI restriction sites. Either promoter alone or promoter with plst-1 coding sequence was injected in smn-1(cb131); ChR2 animals at $30 \mathrm{ng} / \mu \mathrm{l}$ along with the co-injection marker pJM\#67 elt-2p::GFP [64].

Coelomocyte plasmids were created by cloning either PLS3 cDNA or sym-2 RNAi into the unc122p::smn1(RNAi) plasmid using AgeI and NotI. Primers for amplification of sym-2 RNAi were as follows: 5 '-gaacGAATTCTGAGAGGATTGCCTTATGATTGT-3' and 5'-gaacACCGGTctcgatCTATTCCATCAAAATCG-3'. Plasmids were injected into GS1912 at $40 \mathrm{ng} / \mu \mathrm{l}$ alongside coel::RFP (Addgene \#8938) also at $40 \mathrm{ng} / \mu \mathrm{l}$. Transgenic lines tested are listed in Additional file 1: Table 1.

\section{Selection of genes for analysis}

To find proteins in a putative SMN/PLS3 complex, we drew on published pull-down studies in Drosophila S2 cells [45]. We identified proteins that (i) independently pulled down with Fimbrin or Smn with an HG score of more than 61.5 (<0.05 false discovery rate) consistent with "strongly predicted interaction," (ii) were not in the top $10 \%$ of proteins pulled down in the entire database (to avoid non-specific interactions), and (iii) had a C. elegans loss of function allele available in 2011.

\section{C. elegans assays}

Retinal feeding was undertaken as previously described [37] with modifications noted below. Transgenic animals were cultivated in the dark at $20^{\circ} \mathrm{C}$ on NGM plates with OP50 bacteria with or without all-trans retinal (Sigma), for approximately $18 \mathrm{~h}$ before experiments. Plates containing all-trans retinal were prepared by spreading $100 \mu \mathrm{l}$ of OP50 culture mixed with $1.6 \mu \mathrm{l}$ of $100 \mathrm{mM}$ alltrans retinal stock (in ethanol) onto $2.5-\mathrm{cm}$ plates containing $5 \mathrm{ml}$ of NGM. After the animals were picked to individual wells containing PBS and given $5 \mathrm{~min}$ to acclimate to the environment, pre-blue light exposure videos were taken using an AxioCam R3.0 camera at 12 frames/second, on a Zeiss SteREO Discovery V20 microscope at $\times 137.5$ magnification. Immediately following the initial video, animals were illuminated with a mercury bulb and a Zeiss bandpass filter set to $470 \mathrm{~nm}$ to excite channelrhodopsin for $20 \mathrm{~s}$. In order to maintain consistent blue light dosage, light intensity was measured weekly using a spectrometer. To ensure all animals were equally exposed to blue light, a magnification was chosen where the blue light field illuminated well beyond the animals being studied. Post-exhaustion videos were then taken immediately following blue light exposure.

Locomotion analysis was done with young adult animals that were individually picked into a single well of a PDMS chip. Wells within the chip have a diameter of $1.6 \mathrm{~mm}$ and depth of $0.15 \mathrm{~mm}$. Each well was preloaded with $2 \mu \mathrm{l}$ of PBS, and assays were performed at $22^{\circ} \mathrm{C}$. After animals were placed in the wells, they were left undisturbed for 5 min to allow for acclimation to the environment. Locomotion rate was then assessed by taking 10-s videos at relevant time points with an AxioCam R3.0 camera at 12 frames/second, on a Zeiss SteREO Discovery V20 microscope at $\times 137.5$ magnification. 
Movies were analyzed using the NABAS software, modified for image formats, and then translated to body bends per minute [65]. A body bend is defined as a muscle contraction resulting in a dorsal to ventral movement across the midline of the animal. Average body bends per minute $( \pm$ SEM) were combined from at least three independent trials ( $n \geq 30$ animals in total).

Pharyngeal pumping analysis was performed in the last larval stage or in young adults as previously described [39]. Pharyngeal grinder movement in any axis was scored as a pumping event. Average pumping rates $( \pm$ SEM) were combined from at least three independent trials $(n \geq 30$ animals in total).

For locomotion studies, RNAi by feeding was done on second generation (F2) animals reared on either control (pL4440) or experimental, sym-2 [46], hrpf-1, or hrpf-2 dsRNA feeding vectors in HT115 bacteria and assayed. The sym-2 RNAi clone contains genomic DNA inserted into the pL4440 vector, corresponding to the last 3 exons and the 3'UTR of the gene, amplified by primers $5^{\prime}$-CTCGATCTATTCCATCAAAATCG and 5'-TGAGAGGATTGCCTTATGATTGT [66]. $h r p f-1$ and $h r p f-2$ RNAi clones were made by amplifying from genomic DNA using the following primers, respectively, and inserted into the pL4440 vector (5'-ATGGGAGGCC ACTGAACAGG and 5'-GTGATCCATACGCCGCAG AAT; 5'-CGCCCACTGATGTCCGTGG and 5'GCTGGAGATCATCGAAGTGG). RNAi clones were verified by sequencing and aligned for specificity to the gene of interest.

Aldicarb assays were conducted as described [67] with noted modifications. Aldicarb (Sigma-Aldrich) was added to NGM at a final concentration of $1 \mathrm{mM}$. Thirty microliters of OP50 bacteria was seeded in the center of the plates on the day of the assay. The plates were allowed to dry without lids for at least $20 \mathrm{~min}$. Approximately 15 young adult animals were transferred with a platinum wire onto an aldicarb assay plate. The animals were examined every hour and scored as paralyzed if they did not pump or move after being prodded with a platinum wire. The experimenter was blinded to the genotypes/treatment. Average fraction of paralyzed animals $( \pm$ SEM) was obtained by combining at least 3 independent trials ( $n \geq 30$ animals in total).

Coelomocyte endocytic assays were undertaken as described previously [8]. Young adult animals were scored as strong-intense GFP (body cavity filled), some-weak GFP (little GFP in body cavity), or none-no GFP (no GFP visible in the body cavity). Only animals expressing the array in all 6 coelomocytes were scored (determined by the presence of the coelomocyte specific RFP coinjection marker) by an observer blinded to RNAi treatment. Averages were determined over 3 independent trials with an $n>40$.
For the dispersal assay, $60 \times 15 \mathrm{~mm}$ NGM plates were seeded with $200 \mu \mathrm{l}$ of OP50 E. coli and evenly spread over the entire surface. Three rings were drawn on the bottom of the plate, starting $5 \mathrm{~mm}$ from center and every $5 \mathrm{~mm}$ out towards the edge. Strains were maintained at $20^{\circ} \mathrm{C}$ for the duration of the experiment. Approximately 24-32 young adult worms were manually placed in the center of the plate and moved back to $20^{\circ} \mathrm{C}$. Every $60 \mathrm{~min}$ for $180 \mathrm{~min}$, the strains were counted and categorized based on which ring they were in. Animals that moved outside the 3rd ring were counted for total worm count. Data is represented as fractional percent of animals located in each ring.

\section{Preparation of primary murine embryonic fibroblasts (MEFs), cell culture, and transfection}

Primary murine fibroblasts were prepared from E13-14 animals as previously described [16]. Fibroblasts were isolated from $\mathrm{Smn}^{\mathrm{ko} / \mathrm{wt}}$;PLS3V5 ${ }^{\mathrm{tg} / \mathrm{tg}}$ embryos [9].

HEK293T cells and MEFs were grown in culture medium (Dulbecco's modified Eagle's medium (DMEM) supplemented with $10 \%$ fetal calf serum (FCS), $1 \%$ penicillin/streptomycin, and $0.1 \%$ amphotericin) at $37{ }^{\circ} \mathrm{C}$ and $5 \% \mathrm{CO}_{2}$. Eighty percent confluent HEK293T cells were transiently co-transfected with the respective vectors using Lipofectamine 2000 (Invitrogen) and cultured in OptiMEM. Fresh culture medium was added 6-12 h post-transfection to the transfected cells. Cells were harvested $48 \mathrm{~h}$ post-transfection in PBS, and cell pellets were stored at $-80^{\circ} \mathrm{C}$.

\section{Immunofluorescence, immunoblotting, and antibodies for MEFs and HEK293T cells}

For immunofluorescent staining, MEFs were fixed with $4 \%$ paraformaldehyde in PBS for 15-20 min. Fibroblasts were permeabilized in $0.2 \%$ Triton X-100 in PBS for 5 min; blocked for $45 \mathrm{~min}$ in 5\% FCS, $5 \%$ bovine serum albumin (BSA) in PBST; incubated overnight at $4{ }^{\circ} \mathrm{C}$ with the primary antibodies (1:50) in blocking solution; washed with PBS; and incubated with the secondary antibodies (1:200) and phalloidin (1/40) for $3-4 \mathrm{~h}$. For immunofluorescent staining, the following secondary antibodies were used: AlexaFluor 488 (Do anti-Ms 1:300, or Do anti-Rb, 1:300), AlexaFluor 350 (Do anti-Ms 1: 300 , or Do anti-Rb, 1:300), and phalloidin AlexaFluor 568 (Invitrogen, A12380). Cells were imaged using a fluorescence microscope (equipped with an ApoTome (AxioImager M2, Zeiss)). For immunoblotting, the following primary antibodies with the listed concentrations were used: mouse anti-V5 (Invitrogen, R960-25, 1:1000), rabbit anti-hnRNP H2 (Novus Biologicals, NBP1-89816, 1:500), rabbit anti-hnRNP F (Novus Biologicals, NBP157273, 1:500), rabbit anti-PLS3 ([12] 1:1000), anti-SMN (BD Transduction Laboratories, 610647, 1:3000), and 
anti-GFP (produced in hybridoma cell lines, 1:250). For signal detection, the HRP-conjugated secondary antibodies goat anti-mouse (Sigma, A4416, 1:5000) and goat anti-rabbit (Cell Signalling, 7074, 1:5000) lgG were used along with the SuperSignal West Pico Chemiluminescent Substrate (Thermo Scientific). For reprobing, the membranes were stripped for 10 min with the Restore Western Blot Stripping Buffer (Thermo Scientific).

\section{Plasmids}

Human hnRNP $F$ (NM 001098208.1) and hnRNP H2 (NM 019597.4) cDNA was cloned into pcDNA3.1/CTGFP vector and human SMN1 (NM 000344.3) was cloned into pcDNA3.1/V5-His vector via TOPO TA cloning as described in the manufacturer's protocol (Invitrogen). Human PLS3 (NM 005032.6) cDNA was cloned into pcDNA-Flag via MluI and NotI sites. The pcDNA3.1/CT-GFP-TOPO vector (Invitrogen) was used as a negative control for the co-immunoprecipitations.

\section{Co-immunoprecipitation in HEK293T cells}

For GFP-immunoprecipitation (IP), GFP MicroBeads, $\mu$ columns, and $\mu \mathrm{MACS}$ Separator (Miltenyi Biotec) were used. $\mu$-columns were permeabilized with $10 \%$ Triton X100 and washed twice with IP lysis buffer $(100 \mathrm{mM}$ $\mathrm{NaCl}, 50 \mathrm{mM}$ Tris, $5 \mathrm{mM}$ EGTA, 1\% CHAPS, pH 7.5, containing protease inhibitor). Transfected HEK293T cells were lysed for $20 \mathrm{~min}$ on ice in IP lysis buffer, sonicated for $10 \mathrm{~min}$, and centrifuged for $20 \mathrm{~min}$ at 13,000 rpm at $4{ }^{\circ} \mathrm{C}$. Next, $30 \mu \mathrm{l}$ of GFP MicroBeads was added to $500 \mu \mathrm{l}$ cell lysate and incubated for $2 \mathrm{~h}$ at $4{ }^{\circ} \mathrm{C}$. After adding the cell lysates to the $\mu$-columns, the $\mu$-columns were washed 5 times with IP lysis buffer, and proteins were eluted from the $\mu$-column using hot $\left(95^{\circ} \mathrm{C}\right) \mathrm{SDS}$ sample buffer. For RNase treatment, $100 \mu \mathrm{g} / \mathrm{ml}$ RNase A (Invitrogen) was added to the IP lysis buffer. Eluates were analyzed by SDS-PAGE and subsequent Western blot.

\section{Mouse motor neuron differentiation}

Mouse wild-type hb9::GFP ES colonies were differentiated into motor neurons during 6 days and dissociated at day 7 , using an embryoid body method as described previously [68]. Briefly, after 2 days, EBs were treated with retinoic acid (RAc; $100 \mathrm{nM}$, Sigma-Aldrich) and a Hedgehog agonist (500 nM, Curis Inc.). On day 3, EBs were treated with RAc $(100 \mathrm{nM})$ and the Hedgehog agonist $(1 \mu \mathrm{M})$ and incubated for $96 \mathrm{~h}$. On day 7 , papain (Worthington Biochemical) and DNase I were used to dissociate EBs. Motor neurons were then dissociated, plated into poly-ornithine coated greiner 96-well plates (cat \#655090) at a density of 20,000 cells per well, and cultured in DMEM-F12 medium (Invitrogen) containing 2\% FBS (Invitrogen), B-27 supplement (Invitrogen), 20 ng/ml GDNF and BDNF, CNTF (R\&D Systems), insulin, progesterone, BSA, selenite, and apotransferrin (SigmaAldrich).

\section{Immunocytochemistry of primary motor neurons}

Day 13 motor neuron cultures were fixed using 8\% PFA for $15 \mathrm{~min}$, followed by permeabilization and blocking using $0.25 \%$ Triton X-100 and 10\% normal goat serum in PBS for $30 \mathrm{~min}$. Detection of endogenous proteins was performed using the following antibodies: mouse anti-SMN antibody (BD Transduction Laboratories, diluted 1:200), rabbit anti-SMN antibody (Santa Cruz, diluted 1:200), mouse anti-PLS3 antibody (Santa Cruz, diluted 1:500), rabbit anti-hnRNP $\mathrm{H}$ antibody (One World Lab, diluted 1:1000), and rabbit anti-hnRNP F antibody (Abcam, diluted 1:500). Secondary antibodies for detection included AlexaFluor 546 (Gt anti-Rb, 1: 300, or Gt anti-Ms, 1:300) and AlexaFluor 647 (Gt anti$\mathrm{Rb}, 1: 300)$.

\section{Localization analysis}

Images were taken on an Opera system (Perkin Elmer) using a 40x water immersion lens with laser excitation at 405, 488, 561 and $635 \mathrm{nM}$ and used for analysis. Confocal stacks were taken using a Nipkow spinning disk at $1 \mu \mathrm{m}$ slice intervals. Images were stacked together and analyzed for localization of antibody staining using Perkin Elmer's Columbus program (http://www.perkinelmer.com/pages/020/cellularimaging/products/columbus. xhtml).

\section{Co-immunoprecipitation (Co-IP) of mouse brain lysate}

Whole adult mouse brains of Gad2-EGFP mice were prepared by homogenization, using a Polytron homogenizer in $\sim 20 \mathrm{ml}$ of cold 1× RIPA buffer and then incubated on ice for $30 \mathrm{~min}$. Supernatant was collected after a 20-min centrifuge step at $13,500 \mathrm{rpm}$ in $4{ }^{\circ} \mathrm{C}$ and stored at $-80^{\circ} \mathrm{C}$. Protein concentrations were determined using the BSA Protein Assay kit (Bradford).

Seven hundred fifty micrograms of lysate was incubated with $2 \mu \mathrm{g}$ of antibody for $1 \mathrm{~h}$ on a rotator at $4{ }^{\circ} \mathrm{C}$. During incubation, protein $\mathrm{G}$ beads were washed 5 times in PBS and final bead suspension was added to lysate mixture and incubated for $3 \mathrm{~h}$ on a rotator at $4{ }^{\circ} \mathrm{C}$. The bead mixture was centrifuged down and washed three times, removing all supernatant. The beads were then boiled in $2 \times$ SDS loading dye at $95^{\circ} \mathrm{C}$ for 5 min to elute the proteins. Eluted proteins were run on an SDSpolyacrylamide gel for Western analysis.

\section{Western analysis}

Equal amounts of protein preparations were run and separated on a $10 \%$ SDS-polyacrylamide gel electrophoresis and transferred to a PVDF membrane. Membranes 
were blocked with $5 \%$ milk in Tris-buffered saline containing $0.1 \%$ Tween 20 (TBST) overnight at $4{ }^{\circ} \mathrm{C}$. They were probed with the antibody for $1 \mathrm{~h}$ at RT at the following dilutions: mouse anti-SMN antibody (BD Transduction Laboratories, diluted 1:2500), mouse anti-PLS3 antibody (Santa Cruz, diluted 1:1000), rabbit anti-hnRNP$\mathrm{H}$ antibody (One World Lab, diluted 1:10,000), and rabbit anti-hnRNP F antibody (Abcam, diluted 1:2500). After washing with TBST, membranes were incubated with HRP-conjugated secondary antibodies (anti-mouse or anti-rabbit immunoglobulin-horseradish peroxidase, both at 1:2500 dilution) at RT for $30 \mathrm{~min}$, and the blots were developed using ECL and visualized on autoradiography films. The presence of protein was determined based on the size of the band compared to a ladder.

\section{Size exclusion chromatography (SEC)}

HEK293T cells were transfected with hnRNP F-GFP and Flag/His-PLS3 vectors. The size exclusion chromatography was performed on a Superose 6, 10/300 GL column (GE Healthcare) connected to the FPLC system. The SEC column was calibrated with gel filtration standard proteins including thyroglobulin $(669 \mathrm{kDa})$, ferritin $(440 \mathrm{kDa})$, aldolase $(158 \mathrm{kDa})$, ovalbumin $(43 \mathrm{kDa})$, carbonic anhydrase $(29 \mathrm{kDa})$, and ribonuclease A $(13.7 \mathrm{kDa})$. To solubilize proteins including membrane fraction, cells were lysed in the lysis buffer (1\% NP40, $50 \mathrm{mM}$ Tris- $\mathrm{HCl}$ (pH 7.4), 150 $\mathrm{mM} \mathrm{NaCl}$, and $2 \mathrm{mM} \mathrm{MgCl} 2$, containing protease inhibitor cocktail). Lysis buffer without protease inhibitor was degassed and used for column equilibration. The cell lysate was sonicated and centrifuged at 13,000 rpm for 15 min. Two hundred fifty microliters of cell lysate was injected into the column. Fractions of $0.5 \mathrm{ml}$ were collected and frozen at $-80^{\circ} \mathrm{C}$. Twenty-five microliters of each fraction was analyzed by Western blotting.

\section{Supplementary information}

Supplementary information accompanies this paper at https://doi.org/10. 1186/s12915-020-00845-W.

Additional file 1: Figure S1. PLS3 overexpression suppressed pharyngeal pumping defects in $s m n-1$ (ok355) animals. Homozygous null smn-1(ok355) animals lacking smn-1 survive through early larval stages due to maternal loading of SMN-1 proteins and mRNA by heterozygous smn-1 (ok355)/hT2 mothers. hT2 carries a functional copy of endogenous smn-1, overexpression of human PLS3 slightly lowered pumping rates in control $s m n-1(+)$ animals, but increased pumping rates in homozygous smn-1(ok355) animals (compared to control smn-1(ok355)). To control for genetic background all animals were derived from mothers heterozygous for hT2. To control for transgene insertion position, control and smn1(ok355) animals carried rtSiz8 [dpy-30p::empty]. PLS3 is overexpressed from rtSi27 [dpy-30p::PLS3], a single copy insertion on chromosome II. $n \geq 30$ animals per determination, combined from 3 independent trials that the scorer was blinded to the genotype of animals. ANOVA $F(9.9,13.1)=14.23, p<0.001$; post-hoc Mann-Whitney U-test ${ }^{*} p<0.05$, S.E. indicated. Figure S2. sym-2 knockdown using RNAi suppressed the smn1 locomotion defect. Exhaustion of cholinergic motor neurons using ChR2 slowed locomotion; smn-1(cb131) animals with decreased SMN-1 function had aberrantly low locomotion rates post-exhaustion. RNAi knockdown of hnRNP F/H ortholog sym-2 ameliorated this defect. empty (RNAi) used as a control as the bacterial strain used for RNAi can alter locomotion rates. $n \geq 30$ animals per determination, combined from 3 independent trials. Student's $t$-test ${ }^{*} p<0.05$ S.E.M. indicated. Figure S3. Association of SMN and PLS3 with hnRNPF or hnRNPH1/2, is not dependent on RNA. Pretreatment with RNase had no impact on coimmunoprecipitation of PLS3 or SMN from HEK293T cells using GFP tagged hnRNP F or hnRNP H1/2. SMN was tagged with V5; PLS3 was tagged with Flag. Conditions and procedures as described in Fig. 4a. Figure S4.PLS3 and sym-2 suppress behavioral defects in specific C. elegans models of neurodegenerative disease. (a) Neuronal overexpression of wild type human SOD1 (SOD1-WT) had little impact on C. elegans locomotion, but overexpression of SOD1 carrying the familial ALS patient allele G85R (SOD1-G85R) dramatically decreased locomotion in young adult animals. Introduction of ubiquitously expressed human PLS3 decreased locomotion rates in SOD1-WT animals, but ameliorated defects in SOD1-G85R animals. ANOVA $F(320.2,967.5)=6.78, p<0.05$ (b) SOD1-G85R animals had decreased pharyngeal pumping rates in young adult animals. PLS3 expression restored normal pumping rates in SOD1-G85R animals. ANOVA $F(1305379,327194)=235.38, p<0.001$ (c) SOD1-G85R mutant animals were resistant to the cholinesterase inhibitor aldicarb, based on slower time to paralysis. Introduction of ubiquitously expressed human PLS3 restored sensitivity to normal levels. (d) RNAi knockdown of sym-2 had no impact on SOD1-WT animals, but increased locomotion rates in SOD1-G85R animals. Empty RNAi was used as a control for sym-2(RNAi) as bacterial strain can alter locomotion and other behaviors. sym-2(RNAi) had no impact on SOD1-G85R pumping rates (not shown). In this figure PLS3 was ubiquitously expressed from rtSi27 [dpy-30p::PLS3], a single copy insertion on chromosome II. In each assay an $n \geq 30$ animals per determination, combined from 3 independent trials. In all trials scorers were blinded to the genotype of the animals when collecting and scoring the data. MannWhitney U-test (panels a,b,d,e and f) or Log Rank test (panels c and g) ${ }^{* *} p<0.01$ S.E.M. indicated. Figure S5. PLS3 does not generically suppress locomotion. (a) C. elegans lacking glutamic acid decarboxylase encoded by unc-25 had decreased locomotion in liquid due to defective GABA synthesis (Jin et al., 1999). The ubiquitous expression of PLS3 did not suppress locomotion defects in unc-25;PLS3oe double mutant animals. PLS3 was overexpressed from rtSi27 [dpy-30p::PLS3], a single copy insertion on chromosome II. The endogenous C. elegans plastin ortholog, plst-1, was intact. Control single copy insertion rtSi28 did not express PLS3, but was inserted at the same location on $\mathrm{Il}$, confirming that insertion into this locus had no impact on unc- 25 locomotion in this assay. $n \geq 30$ animals per determination, combined from 3 independent trials. ANOVA $F(7.37,7.45)=20.09, p<0.001$ Mann-Whitney U-test: ${ }^{* *} p<0.01$, S.E.M indicated. b) PLS3 overexpression is unable to rescue the unc phenotype in unc-13 animals when assessing dispersal on food. Animals are reported as the percentage of animals that escaped the center ring after 1,2 or 3 hours. Mean percent of animals escaped is calculated from three independent trials of at least 24 animals per genotype, per trial. Each trial is shown as a grey circle. Figure S6. Uncropped western blots. Panels a, d and e correspond to Fig. 5 panels $\mathrm{a}, \mathrm{b}$ and $\mathrm{c}$, respectively. Panels $\mathrm{b}$ and $\mathrm{c}$ correspond to supplementary figure 3 . Table S1. Strains.

\section{Acknowledgements}

We thank members of the Hart, Rubin, Artavanis-Tsakonas, Van Vactor, and Wirth laboratory for helpful discussions. We thank the Morimoto and Wang lab for providing PolyQ and ALS models, respectively, and the ArtavanisTsakonas lab for providing immunoprecipitation results prior to publication.

\section{Authors' contributions}

MW and $\mathrm{AH}$ designed the experiments and wrote the manuscript, with contributions from EJ, MH, and BW. Most experiments were undertaken by MW, including C. elegans experiments, motor neuron staining, mouse brain co-immunoprecipitation, bioinformatics, and analysis. EW assisted with the $C$. elegans genetics and behavioral analysis of ALS and polyQ models. MD assisted with the cloning experiments. MH performed the spinal cord fractionations, and EJ performed the work done in HEK293T cells and mouse fibroblast cells. NM, LD, and EM provided help with the motor neuron 
differentiation and localization analysis. All authors reviewed and approved the manuscript.

\section{Funding}

Some strains were provided by the C. elegans Genetics Center (CGC), which is funded by NIH Office of Research Infrastructure Programs (P40 OD010440). We also thank J. Kauer for murine tissue samples. This study was funded by the NIH-NINDS P01 to ACH and grants from the Deutsche Forschungsgemeinschaft Wi-945/16-1, Wi 945/17-1, Wi-945/18-1 and RTG 1960, and the Center for Molecular Medicine Cologne (grant no. C16) to BW.

\section{Availability of data and materials}

New C. elegans strains generated here are available from the Hart laboratory and/or the CGC. Plasmids are available from the Hart laboratory. Data supporting all of the figures will be made available at a permanent DO location via Brown University upon publication.

\section{Ethics approval and consent to participate}

Not applicable for C. elegans studies. Animal care and all surgical procedures were performed according to the institutional animal care committee guidelines and the German animal welfare laws and approved under the reference numbers 84-02.04.2014.A006 and 84-02.04.2015.A378 of the LANUV state agency of North Rhine-Westphalia.

\section{Consent for publication}

Not applicable.

\section{Competing interests}

The authors declare that they have no competing interests.

\section{Author details}

'Department of Neuroscience, Brown University, 185 Meeting Street, Mailbox GL-N, Providence, RI 02912, USA. ${ }^{2}$ Institute of Human Genetics, Center for Molecular Medicine Cologne, Institute of Genetics, and Center for Rare Disorders, University of Cologne, Cologne, Germany. ${ }^{3}$ Department of Stem Cell \& Regenerative Biology, Harvard University, Cambridge, MA 02138, USA. ${ }^{4}$ Department of Biological and Environmental Sciences, University of Hertfordshire, Hertfordshire, UK.

\section{Received: 31 March 2020 Accepted: 11 August 2020 Published online: 16 September 2020}

\section{References}

1. Fischer U, Liu Q, Dreyfuss G. The SMN-SIP1 complex has an essential role in spliceosomal snRNP biogenesis. Cell. 1997;90(6):1023-9.

2. Liu Q, Fischer U, Wang F, Dreyfuss G. The spinal muscular atrophy disease gene product, SMN, and its associated protein SIP1 are in a complex with spliceosomal snRNP proteins. Cell. 1997;90(6):1013-21.

3. Pellizzoni L, Yong J, Dreyfuss G. Essential role for the SMN complex in the specificity of snRNP assembly. Science. 2002;298(5599):1775-9.

4. Zou T, Yang X, Pan D, Huang J, Sahin M, Zhou J. SMN deficiency reduces cellular ability to form stress granules, sensitizing cells to stress. Cell Mol Neurobiol. 2011;31(4):541-50.

5. Hua Y, Zhou J. Rpp20 interacts with SMN and is re-distributed into SMN granules in response to stress. Biochem Biophys Res Commun. 2004;314(1): 268-76.

6. Fallini $\mathrm{C}$, Zhang HL, Su YH, Silani V, Singer RH, Rossoll W, Bassell GJ. The survival of motor neuron (SMN) protein interacts with the mRNA-binding protein $\mathrm{HuD}$ and regulates localization of poly(A) mRNA in primary motor neuron axons. J Neurosci. 2011;31(10):3914-25.

7. Akten B, Kye MJ, Hao LT, Wertz MH, Singh S, Nie DY, Huang J, Merianda TT, Twiss JL, Beattie CE, et al. Interaction of survival of motor neuron (SMN) and HuD proteins with mRNA cpg15 rescues motor neuron axonal deficits. P Natl Acad Sci USA. 2011;108(25):10337-42.

8. Dimitriadi M, Derdowski A, Kalloo G, Maginnis MS, O'Hern P, Bliska B, Sorkac A, Nguyen KC, Cook SJ, Poulogiannis G, et al. Decreased function of survival motor neuron protein impairs endocytic pathways. Proc Natl Acad Sci U S A. 2016;113(30):E4377-86.

9. Hosseinibarkooie S, Peters M, Torres-Benito L, Rastetter RH, Hupperich K, Hoffmann A, Mendoza-Ferreira N, Kaczmarek A, Janzen E, Milbradt J, et al. The power of human protective modifiers: PLS3 and CORO1C unravel impaired endocytosis in spinal muscular atrophy and rescue SMA phenotype. Am J Hum Genet. 2016;99(3):647-65.

10. Riessland M, Kaczmarek A, Schneider S, Swoboda K, Lohr H, Bradler C, Grysko V, Dimitriadi M, Hosseinibarkooie S, Torres-Benito L, et al. Neurocalcin delta suppression protects against spinal muscular atrophy in humans and across species by restoring impaired endocytosis. Am J Hum Genet. 2017;100(2):297-315.

11. O'Hern PJ, do Carmo GGI, Brecht J, Lopez Soto EJ, Simon J, Chapkis N, Lipscombe D, Kye MJ, Hart AC: Decreased microRNA levels lead to deleterious increases in neuronal M2 muscarinic receptors in Spinal Muscular Atrophy models. Elife. 2017;6:e20752. https://doi.org/10.7554/eLife. 20752.

12. Oprea GE, Krober S, McWhorter ML, Rossoll W, Muller S, Krawczak M, Bassell GJ, Beattie CE, Wirth B. Plastin 3 is a protective modifier of autosomal recessive spinal muscular atrophy. Science. 2008:320(5875):524-7.

13. Delanote V, Vandekerckhove J, Gettemans J. Plastins: versatile modulators of actin organization in (patho) physiological cellular processes. Acta Pharmacol Sin. 2005;26(7):769-79.

14. Engqvist-Goldstein AE, Drubin DG. Actin assembly and endocytosis: from yeast to mammals. Annu Rev Cell Dev Biol. 2003;19:287-332.

15. McGovern VL, Massoni-Laporte A, Wang X, Le TT, Le HT, Beattie CE, Rich MM, Burghes AH. Plastin 3 expression does not modify spinal muscular atrophy severity in the 7 SMA mouse. PLoS One. 2015;10(7):e0132364.

16. Ackermann B, Krober S, Torres-Benito L, Borgmann A, Peters M, Barkooie SMH, Tejero R, Jakubik M, Schreml J, Milbradt J, et al. Plastin 3 ameliorates spinal muscular atrophy via delayed axon pruning and improves neuromuscular junction functionality. Hum Mol Genet. 2013;22(7):1328-47.

17. Alrafiah A, Karyka E, Coldicott I, Iremonger K, Lewis KE, Ning K, Azzouz M. Plastin 3 promotes motor neuron axonal growth and extends survival in a mouse model of spinal muscular atrophy. Mol Ther Methods Clin Dev. 2018;9:81-9.

18. Kaifer KA, Villalón E, Osman EY, Glascock JJ, Arnold LL, DDW C, Lorson CL. Plastin-3 extends survival and reduces severity in mouse models of spinal muscular atrophy. JCl Insight. 2017;2(5):e89970.

19. Janzen E, Mendoza-Ferreira N, Hosseinibarkooie S, Schneider S, Hupperich K Tschanz T, Grysko V, Riessland M, Hammerschmidt M, Rigo F, Bennett CF, Kye MJ, Torres-Benito L, Wirth B. CHP1 reduction ameliorates spinal muscular atrophy pathology by restoring calcineurin activity and endocytosis. Brain. 2018;141(8):2343-61.

20. Janzen E, Wolff L, Mendoza-Ferreira N, Hupperich K, DelleVedove A, Hosseinibarkooie S, Kye MJ, Wirth B. PLS3 overexpression delays ataxia in Chp1 mutant mice. Front Neurosci. 2019;13:993.

21. Cooper TA, Wan L, Dreyfuss G. RNA and disease. Cell. 2009;136:777-93.

22. Khalil B, Morderer D, Price PL, Liu F, Rossoll W. mRNP assembly, axonal transport, and local translation in neurodegenerative diseases. Brain Res. 2018;1693(Pt A):75-91.

23. Liu-Yesucevitz L, Bassell GJ, Gitler AD, Hart AC, Klann E, Richter JD, Warren ST, Wolozin B. Local RNA translation at the synapse and in disease. Neurosci. 2011;31(45):16086-93

24. Nizzardo M, Taiana M, Rizzo F, Aguila Benitez J, Nijssen J, Allodi I, Melzi V, Bresolin N, Comi GP, Hedlund E, Corti S. Synaptotagmin 13 is neuroprotective across motor neuron diseases. Acta Neuropathol. 2020.

25. Hao le, T., H. R. Fuller, T. Lam le, T. T. Le, A. H. Burghes and G. E. Morris: Absence of gemin5 from SMN complexes in nuclear Cajal bodies. BMC Cell Biol 2007. 8: 28.

26. Cacciottolo R, Ciantar J, Lanfranco M, Borg RM, Vassallo N, Bordonne R, Cauchi RJ. SMN complex member Gemin3 self-interacts and has a functional relationship with ALS-linked proteins TDP-43, FUS and Sod1. Sci Rep. 2019;9(1):18666.

27. Gertz B, Wong M, Martin LJ. Nuclear localization of human SOD1 and mutant SOD1-specific disruption of survival motor neuron protein complex in transgenic amyotrophic lateral sclerosis mice. J Neuropathol Exp Neuro. 2012;71(2):162-77.

28. Kariya S, Re DB, Jacquier A, Nelson K, Przedborski S, Monani UR. Mutant superoxide dismutase 1 (SOD1), a cause of amyotrophic lateral sclerosis, disrupts the recruitment of SMN, the spinal muscular atrophy protein to nuclear Cajal bodies. Hum Mol Genet. 2012;21(15):3421-34.

29. Yanagi KS, Wu Z, Amaya J, Chapkis N, Duffy AM, Hajdarovic KH, Held A, Mathur AD, Russo K, Ryan VH, Steinert BL, Whitt JP, Fallon JR, Fawzi NL, Lipscombe D, Reenan RA, Wharton KA, Hart AC. Meta-analysis of genetic modifiers reveals candidate dysregulated pathways in amyotrophic lateral sclerosis. Neuroscience. 2019;396:A3-a20. 
30. Wang J, Farr GW, Hall DH, Li F, Furtak K, Dreier L, Horwich AL: An ALS-linked mutant SOD1 produces a locomotor defect associated with aggregation and synaptic dysfunction when expressed in neurons of Caenorhabditis elegans. PLoS Genet. 2009;5(1):e1000350. https://doi.org/10.1371/journal. pgen.1000350

31. Briese M, Esmaeili B, Fraboulet S, Burt EC, Christodoulou S, Towers PR, Davies KE, Sattelle DB. Deletion of smn-1, the Caenorhabditis elegans ortholog of the spinal muscular atrophy gene, results in locomotor dysfunction and reduced lifespan. Hum Mol Genet. 2009;18(1):97-104.

32. Miguel-Aliaga I, Culetto E, Walker DS, Baylis HA, Sattelle DB, Davies KE. The Caenorhabditis elegans orthologue of the human gene responsible for spinal muscular atrophy is a maternal product critical for germline maturation and embryonic viability. Hum Mol Genet. 1999;8(12):2133-43.

33. Sleigh JN, Buckingham SD, Cuppen E, Viswanathan M, Westlund BM, Sattelle DB. A novel point mutation in the Caenorhabditis elegans smn-1 gene provides a useful model for investigating Spinal Muscular Atrophy. Neuromuscul Disord. 2010:20:S22-3.

34. Sun Y, Grimmler M, Schwarzer V, Schoenen F, Fischer U, Wirth B. Molecular and functional analysis of intragenic SMN1 mutations in patients with spinal muscular atrophy. Hum Mutat. 2005;25(1):64-71.

35. Sleigh JN, Buckingham SD, Esmaeili B, Viswanathan M, Cuppen E, Westlund BM, Sattelle DB. A novel Caenorhabditis elegans allele, smn-1(cb131), mimicking a mild form of spinal muscular atrophy, provides a convenient drug screening platform highlighting new and pre-approved compounds. Hum Mol Genet. 2011;20(2):245-60.

36. Liewald JF, Brauner M, Stephens GJ, Bouhours M, Schultheis C, Zhen M, Gottschalk A. Optogenetic analysis of synaptic function. Nat Methods. 2008; 5(10):895-902

37. Liu Q, Hollopeter G, Jorgensen EM. Graded synaptic transmission at the Caenorhabditis elegans neuromuscular junction. P Natl Acad Sci USA. 2009; 106(26):10823-8.

38. Hao LT, Wolman M, Granato $M$, Beattie CE. Survival motor neuron affects plastin 3 protein levels leading to motor defects. J Neurosci. 2012;32(15):5074-84.

39. Dimitriadi M, Sleigh JN, Walker A, Chang HC, Sen A, Kalloo G, Harris J, Barsby T, Walsh MB, Satterlee JS, Li C, Van Vactor D, Artavanis-Tsakonas S, Hart AC. Conserved genes act as modifiers of invertebrate SMN loss of function defects. PLoS genetics. 2010;6(10):e1001172. https://doi.org/10.1371/journal. pgen.1001172.

40. Sen A, Dimlich DN, Guruharsha KG, Kankel MW, Hori K, Yokokura T, Brachat S, Richardson D, Loureiro J, Sivasankaran R, et al. Genetic circuitry of survival motor neuron, the gene underlying spinal muscular atrophy. P Natl Acad Sci USA. 2013;110(26):E2371-80.

41. Hsu DR, Chuang PT, Meyer BJ. DPY-30, a nuclear protein essential early in embryogenesis for Caenorhabditis elegans dosage compensation. Development. 1995:121(10):3323-34.

42. Dreyfuss G, Kim VN, Kataoka N. Messenger-RNA-binding proteins and the messages they carry. Nat Rev Mol Cell Bio. 2002;3(3):195-205.

43. Dearruda MV, Watson S, Lin CS, Leavitt J, Matsudaira P. Fimbrin is a homolog of the cytoplasmic phosphoprotein plastin and has domains homologous with calmodulin and actin gelation proteins. J Cell Biol. 1990; 111(3):1069-79.

44. Sandrock TM, Brower SM, Toenjes KA, Adams AEM. Suppressor analysis of fimbrin (Sac6p) overexpression in yeast. Genetics. 1999:151(4):1287-97.

45. Guruharsha KG, Rual JF, Zhai B, Mintseris J, Vaidya P, Vaidya N, Beekman C, Wong C, Rhee DY, Cenaj O, McKillip E, Shah S, Stapleton M, Wan KH, Yu C, Parsa B, Carlson JW, Chen X, Kapadia B, Vijayraghavan K, Gygi SP, Celniker SE, Obar RA, Artavanis-Tsakonas S. A protein complex network of Drosophila melanogaster. Cell. 2011;147(3):690-703.

46. Yochem J, Bell LR, Herman RK. The identities of sym-2, sym-3 and sym-4, three genes that are synthetically lethal with mec-8 in Caenorhabditis elegans. Genetics. 2004;168(3):1293-306.

47. Dreyfuss G, Matunis MJ, Pinolroma S, Burd CG. Hnrnp proteins and the biogenesis of messenger-Rna. Annu Rev Biochem. 1993;62:289-321.

48. Liu Y, Gervasi C, Szaro BG. A crucial role for hnRNP K in axon development in Xenopus laevis. Development. 2008;135(18):3125-35.

49. Pagliardini S, Giavazzi A, Setola V, Lizier C, Di Luca M, DeBiasi S, Battaglia G. Subcellular localization and axonal transport of the survival motor neuron (SMN) protein in the developing rat spinal cord. Hum Mol Genet. 2000;9(1): 47-56.

50. Rossoll W, Kroning AK, Ohndorf UM, Steegborn C, Jablonka S, Sendtner M. Specific interaction of Smn, the spinal muscular atrophy determining gene product, with hnRNP-R and gry-rbp/hnRNP-Q: a role for Smn in RNA processing in motor axons? Hum Mol Genet. 2002;11(1):93-105.

51. Gupta SK, Kosti I, Plaut G, Pivko A, Tkacz ID, Cohen-Chalamish S, Biswas DK, Wachtel C, Ben-Asher HW, Carmi S, et al. The hnRNP F/H homologue of Trypanosoma brucei is differentially expressed in the two life cycle stages of the parasite and regulates splicing and mRNA stability. Nucleic Acids Res. 2013:41(13):6577-94.

52. Wang E, Aslanzadeh V, Papa F, Zhu H, de la Grange P, Cambi F. Global profiling of alternative splicing events and gene expression regulated byhnRNPH/F. PLoS One. 2012;7(12):e51266. https://doi.org/10.1371/journal. pone.0051266.

53. Fitzgerald K, Greenwald I. Interchangeability of Caenorhabditis elegans DSL proteins and intrinsic signalling activity of their extracellular domains in vivo. Development. 1995;121(12):4275-82.

54. Grant B, Greenwald I. Structure, function, and expression of SEL-1, a negative regulator of LIN-12 and GLP-1 in C. elegans. Development. 1997; 124(3):637-44.

55. Fares H, Greenwald I. Genetic analysis of endocytosis in Caenorhabditis elegans: coelomocyte uptake defective mutants. Genetics. 2001;159(1):13345.

56. Nishimura AL, Mitne-Neto M, Silva HCA, Richieri-Costa A, Middleton S, Cascio D, Kok F, Oliveira JRM, Gillingwater T, Webb J, et al. A mutation in the vesicle-trafficking protein VAPB causes late-onset spinal muscular atrophy and amyotrophic lateral sclerosis. Am J Hum Genet. 2004;75(5):82231.

57. Bastow E, Peswani AR, Tarrant D, Pentland DR, Chen X, Morgan A, Staniforth G, Tullet JM, Rowe ML, Howard MF, et al. New links between SOD1 and metabolic dysfunction from a yeast model of amyotrophic lateral sclerosis. J Cell Sci. 2016;129:4118-29.

58. Jin YS, Jorgensen E, Hartwieg E, Horvitz HR. The Caenorhabditis elegans gene unc-25 encodes glutamic acid decarboxylase and is required for synaptic transmission but not synaptic development. J Neurosci. 1999;19(2): 539-48.

59. Yu A, Shibata Y, Shah B, Calamini B, Lo DC, Morimoto RI. Protein aggregation can inhibit clathrin-mediated endocytosis by chaperone competition. Proc Natl Acad Sci U S A. 2014;111(15):E1481-90.

60. Urwin H, Authier A, Nielsen JE, Metcalf D, Powell C, Froud K, Malcolm DS, Holm I, Johannsen P, Brown J, et al. Disruption of endocytic trafficking in frontotemporal dementia with CHMP2B mutations. Hum Mol Genet. 2010; 19(11):2228-38.

61. Brenner S. Genetics of Caenorhabditis-elegans. Genetics. 1974;77(1):71-94

62. Calixto A, Chelur D, Topalidou I, Chen XY, Chalfie M. Enhanced neuronal RNAi in C. elegans using SID-1. Nat Methods. 2010;7(7):554-U102.

63. Frokjaer-Jensen C, Davis MW, Hopkins CE, Newman BJ, Thummel JM, Olesen SP, Grunnet M, Jorgensen EM. Single-copy insertion of transgenes in Caenorhabditis elegans. Nat Genet. 2008:40(11):1375-83.

64. Fukushige T, Hawkins MG, McGhee JD. The GATA-factor elt-2 is essential for formation of the Caenorhabditis elegans intestine. Dev Biol. 1998;198(2): 286-302.

65. Krajacic $P$, Shen XN, Purohit PK, Arratia P, Lamitina T. Biomechanical profiling of Caenorhabditis elegans motility. Genetics. 2012;191(3):1015-U1613.

66. Kamath RS, Fraser AG, Dong Y, Poulin G, Durbin R, Gotta M, Kanapin A, Le Bot N, Moreno S, Sohrmann M, et al. Systematic functional analysis of the Caenorhabditis elegans genome using RNAi. Nature. 2003;421(6920):231-7.

67. Mahoney TR, Luo S, Nonet ML. Analysis of synaptic transmission in Caenorhabditis elegans using an aldicarb-sensitivity assay. Nat Protoc. 2006; 1(4):1772-7.

68. Wichterle H, Lieberam I, Porter JA, Jessell TM. Directed differentiation of embryonic stem cells into motor neurons. Cell. 2002;110(3):385-97.

\section{Publisher's Note}

Springer Nature remains neutral with regard to jurisdictional claims in published maps and institutional affiliations. 